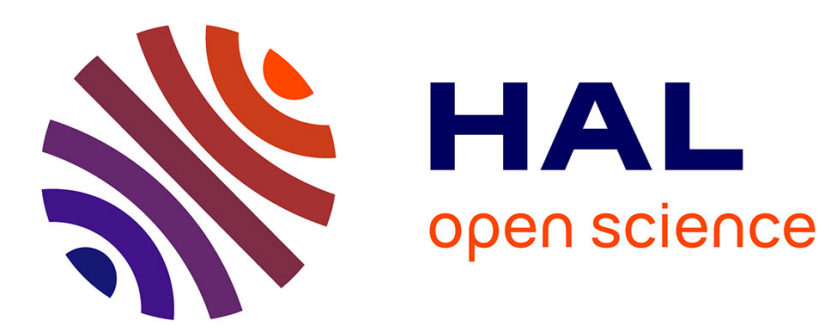

\title{
Analysis of the null-controllability of degenerate parabolic systems of Grushin type via the moments method
}

\author{
Damien Allonsius, Franck Boyer, Morgan Morancey
}

\section{- To cite this version:}

Damien Allonsius, Franck Boyer, Morgan Morancey. Analysis of the null-controllability of degenerate parabolic systems of Grushin type via the moments method. Journal of Evolution Equations, 2021, 21, pp.4799-4843. hal-02471592

\section{HAL Id: hal-02471592 \\ https://hal.science/hal-02471592}

Submitted on 8 Feb 2020

HAL is a multi-disciplinary open access archive for the deposit and dissemination of scientific research documents, whether they are published or not. The documents may come from teaching and research institutions in France or abroad, or from public or private research centers.
L'archive ouverte pluridisciplinaire HAL, est destinée au dépôt et à la diffusion de documents scientifiques de niveau recherche, publiés ou non, émanant des établissements d'enseignement et de recherche français ou étrangers, des laboratoires publics ou privés. 


\title{
ANALYSIS OF THE NULL-CONTROLLABILITY OF DEGENERATE PARABOLIC SYSTEMS OF GRUSHIN TYPE VIA THE MOMENTS METHOD
}

\author{
D. ALLONSIUS*, F. BOYER ${ }^{\dagger}$, AND M. MORANCEY*
}

\begin{abstract}
In this article we compute the exact value of the minimal null control time for the Grushin equation controlled on a strip. This result is already known from a recent work by K. Beauchard, J. Dardé and S. Ervedoza but we propose a different approach based on the moments method. Our approach requires a careful spectral analysis of a truncated harmonic oscillator. We have also extended known results on biorthogonal families to real exponentials in the absence of a gap condition in order to obtain uniform estimates with respect to the asymptotic beahviour of the associated counting function. As a byproduct of our approach we also characterize the minimal null control time for systems of coupled Grushin equations with a distributed control on a strip or with a boundary control.
\end{abstract}

Key words. Control theory; degenerate parabolic partial differential equations; minimal null control time; moments method; biorthogonal families; spectral analysis of harmonic oscillator.

AMS subject classifications. 93B05; 93C20; 30E05; 35P20

\section{Introduction.}

\subsection{The problem under study.}

Let $T>0, \Omega:=(-1,1) \times(0,1)$ and $\omega$ be an open subset of $\Omega$. Let $\mathcal{D}$ be the degenerate elliptic operator $\mathcal{D}:=-\partial_{x x}-x^{2} \partial_{y y}$. We are interested in the distributed null-controllability properties of the associated parabolic equation (Grushin's equation) with Dirichlet boundary condition, that reads

$$
\begin{cases}\partial_{t} f(t, x, y)+\mathcal{D} f(t, x, y)=1_{\omega} u(t, x, y), & (t, x, y) \in(0, T) \times \Omega \\ f(t, x, y)=0, & (t, x, y) \in(0, T) \times \partial \Omega \\ f(0, x, y)=f^{0}(x, y) \in L^{2}(\Omega), & (x, y) \in \Omega .\end{cases}
$$

More precisely, given $T>0$ we will say that 1.1 is null controllable at time $T$ if for any $f^{0} \in L^{2}(\Omega)$ there exists a control $u \in L^{2}((0, T) \times \Omega)$ such that $\forall(x, y) \in$ $\Omega, f(T, x, y)=0$.

It turns out that when $\omega=(a, b) \times(0,1)$ where $0<a<b \leq 1$, see Figure 1.1 . there exists a positive minimal null control time, that is, a time $T^{*}>0$ such that

- when $T \leq T^{*}$, equation 1.1 is not null controllable at time $T$,

- when $T>T^{*}$, equation (1.1) is null controllable at time $T$.

More precisely, it was proved in [6] that $T^{*} \geq \frac{a^{2}}{2}$ and, very recently, the following sharp result was obtained in [7].

THEOREM 1.1. Let $0<a<b \leq 1$. The minimal null control time of (1.1) with $\omega=(a, b) \times(0,1)$ is exactly given by

$$
T^{*}=\frac{a^{2}}{2}
$$

\footnotetext{
*Aix-Marseille Université, CNRS, Centrale Marseille, I2M, UMR 7373, 13453 Marseille, France (damien.allonsius@univ-amu.fr, morgan.morancey@univ-amu.fr).

${ }^{\dagger}$ Institut de Mathématiques de Toulouse \& Institut Universitaire de France, UMR 5219, Université de Toulouse, CNRS, UPS IMT, F-31062 Toulouse Cedex 9, France (franck.boyer@math. univ-toulouse.fr)
} 


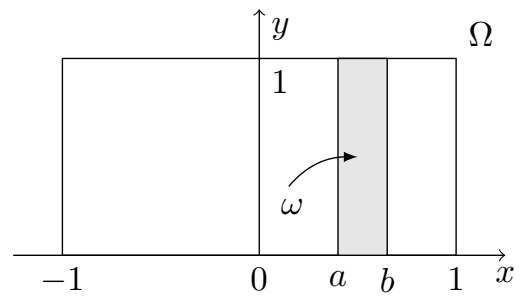

Figure 1.1: The geometrical setting

The first motivation of our work is to give another proof of this result using moments method. We will take the opportunity of this work to develop new estimates for biorthogonal sequences to real exponentials (see Theorem 1.3) that may have a wider range of applications. Our approach will also enable us to treat coupled systems of such Grushin equations (see Theorem 1.4).

Let $\Gamma_{1}=\{1\} \times[0,1]$. With classical arguments (see [18, Appendix A] for details in this setting), based on extensions of the spatial domain and cut-off functions, it directly comes from Theorem 1.1 that the minimal null control time for the boundary control problem

$$
\begin{cases}\partial_{t} f(t, x, y)+\mathcal{D} f(t, x, y)=0, & (t, x, y) \in(0, T) \times \Omega \\ f(t, x, y)=0, & (t, x, y) \in(0, T) \times\left(\partial \Omega \backslash \Gamma_{1}\right) \\ f(t, x, y)=u(t, y), & (t, x, y) \in(0, T) \times \Gamma_{1} \\ f(0, x, y)=f^{0}(x, y) \in L^{2}(\Omega), & (x, y) \in \Omega,\end{cases}
$$

is given by $T^{*}=\frac{1}{2}$. We will prove directly this result since it extends at no cost to coupled systems of such Grushin equations (see Theorem 1.5) with a single boundary control. To the best of our knowledge, this result is out of reach with other techniques.

\subsection{State of the art.}

The counter-example proving that (1.1) is not null controllable in time $T \leq \frac{a^{2}}{2}$ has been exhibited in the pioneering work [6, Section 3.3]. We will thus focus on the positive null controllability result in time $T>\frac{a^{2}}{2}$. In 8 the value of the minimal null control time $T^{*}$ has been proved to be exactly $\frac{a^{2}}{2}$ when the control domain is symmetric with respect to the degeneracy i.e. $\omega=((-b,-a) \cup(a, b)) \times(0,1)$. In [7, it has been recently proved that $T^{*}:=\frac{a^{2}}{2}$ also when $\omega=(a, b) \times(0,1)$, that is Theorem 1.1. We mention that the results obtained in [7] cover more general situations than the one considered in Theorem 1.1 non symmetric space domains, higher dimension (in the $y$ variable), Dirichlet or Neumann controls and more general degeneracies.

Let us mention that, as detailed in [5, Section 4.1.3], the reason for the appearance of such a minimal time, is the insufficient observation of some eigenvectors of $\mathcal{D}$ that localizes near the singular set $\{x=0\}$ too quickly with respect to the desired control time.

The appearance of a minimal null control time in this context is very much related to the geometry of $\omega$. Indeed, it is proved in 25] that if $\Omega \backslash \omega$ contains an horizontal strip $(-1,1) \times(a, b)$, then equation 1.1$)$ is not null controllable whatever the time $T$ is. 
The proof is done by contradiction using for instance Runge's approximation theorem with quite subtle estimates coming from complex analysis. An (almost) complete study of the value of the minimal null control time with more complex geometries for $\omega$ is done in [18.

The common point between the results obtained in [6, 7, 8, and the analysis proposed in this article is to take advantage of the cartesian geometry of $\Omega$ and $\omega$ to reduce the study to the one of a family of one dimensional problems. To be more precise, let us introduce the operators

$$
\mathcal{A}_{n}:=-\partial_{x x}+(n \pi x)^{2}, \quad D\left(\mathcal{A}_{n}\right):=H^{2}(-1,1) \cap H_{0}^{1}(-1,1),
$$

and let $\phi_{n}$ be the $n^{\text {th }}$ normalized eigenfunction of Dirichlet Laplace operator on $(0,1)$ :

$$
\phi_{n}(y):=\sqrt{2} \sin (n \pi y), \quad y \in(0,1) .
$$

Then, as proved in [8, Section 2.3], for any $u \in L^{2}((0, T) \times \omega)$, expanding

$$
u(t, x, y)=\sum_{n \in \mathbb{N}^{*}} u_{n}(t, x) \phi_{n}(y)
$$

we get that the solution $f$ of 1.1 can be written as

$$
f(t, x, y)=\sum_{n \in \mathbb{N}^{*}} f_{n}(t, x) \phi_{n}(y),
$$

where $f_{n}$ is the solution of the following one dimensional non-degenerate parabolic equation

$$
\begin{cases}\partial_{t} f_{n}(t, x)+\mathcal{A}_{n} f_{n}(t, x)=u_{n}(t, x) 1_{(a, b)}(x), & (t, x) \in(0, T) \times(-1,1), \\ f_{n}(t, \pm 1)=0, & t \in(0, T), \\ f_{n}(0, x)=f_{n}^{0}(x), & x \in(-1,1) .\end{cases}
$$

The null controllability of (1.4) is well known (see for instance [20]) and holds for any $T>0$. Thus, proving null controllability of (1.1) amounts to estimate a control $u_{n}$ realizing null controllability of (1.4) with respect to $n$. Indeed, if for a given $T>0$, the cost of null controllability of 1.4 is bounded with respect to $n$ i.e. if there exists $C>0$ such that for any $n$ and any $f_{n}^{0} \in L^{2}(-1,1)$ there exists a control $u_{n} \in L^{2}((0, T) \times(a, b))$ satisfying

$$
\left\|u_{n}\right\|_{L^{2}((0, T) \times(a, b))} \leq C\left\|f_{n}^{0}\right\|_{L^{2}(-1,1)}
$$

we will get that 1.3 actually defines a control $u \in L^{2}((0, T) \times \omega)$ for our initial problem and thus gives null controllability of 1.1 in time $T$.

In [6, 7, the study of the cost of controllability of (1.4), or equivalently the cost of observability of the adjoint problem, is done using Carleman estimates with adapted weights. In [8], this analysis relies on the transmutation that reduces the problem to estimating the cost of observability of the wave equation associated with $\mathcal{A}_{n}$. This study is then done, as the equation is one dimensional, using sideways energy estimates (i.e. energy estimates where the role of the time and space variables are interchanged).

In this article we propose a new strategy, based on the the moments method, allowing to recover Theorem 1.1 that we describe in the next section. 


\subsection{Our method.}

As already mentioned, we reduced our problem to the one of proving that, when $T>\frac{a^{2}}{2}$, the cost of null-controllability of 1.4 is bounded with respect to $n$. We will achieve this objective by using the moments method, that is why we first recall the construction of a null control of 1.4 with this strategy.

For any $n \geq 1$, we denote by $\Lambda_{n}:=\left(\lambda_{k, n}\right)_{k \geq 1}$ the increasing sequence of eigenvalues of the operator $\mathcal{A}_{n}$. We also denote by $\left(g_{k, n}\right)_{k>1}$ the associated Hilbert basis of $L^{2}(-1,1)$ of eigenfunctions and by $e^{-\cdot \mathcal{A}_{n}}$ the semigroup generated by $-\mathcal{A}_{n}$.

Let $u_{n} \in L^{2}((0, T) \times(a, b))$ and $f_{n}$ be a solution of (1.4). Testing the equation against the solution $t \mapsto e^{-(T-t) \mathcal{A}_{n}} g_{k, n}=e^{-(T-t) \lambda_{k, n}} g_{k, n}$ of the backward problem, we obtain the following identity

$$
\begin{aligned}
\int_{0}^{T}\left\langle u_{n}(t, \cdot), e^{-\lambda_{k, n}(T-t)} g_{k, n}\right\rangle_{L^{2}(a, b)} \mathrm{d} t= & \left\langle f_{n}(T, \cdot), g_{k, n}\right\rangle_{L^{2}(-1,1)} \\
& -\left\langle f_{n}^{0}, e^{-\lambda_{k, n} T} g_{k, n}\right\rangle_{L^{2}(-1,1)} .
\end{aligned}
$$

As $\left(g_{k, n}\right)_{k \geq 1}$ is an Hilbert basis of $L^{2}(-1,1)$, it comes that $f_{n}(T, \cdot)=0$ if and only if the control $u_{n}$ satisifes the following moments problem: for any $k \in \mathbb{N}^{*}$,

$$
\int_{0}^{T}\left\langle u_{n}(t, \cdot), e^{-\lambda_{k, n}(T-t)} g_{k, n}\right\rangle_{L^{2}(a, b)} \mathrm{d} t=-\left\langle f_{n}^{0}, e^{-\lambda_{k, n} T} g_{k, n}\right\rangle_{L^{2}(-1,1)} .
$$

Following for instance [27, Theorem 4], it comes that for any $n \in \mathbb{N}^{*}, \sum_{k \in \mathbb{N}^{*}} \frac{1}{\lambda_{k, n}}<$ $+\infty$. Thus, using for instance [28, $\S 7$ and $\S 12]$, we have that, for any $n \geq 1$, there exists a biorthogonal family to the exponential functions associated to $\Lambda_{n}$ i.e. a family $\left(q_{j, n}\right)_{j \in \mathbb{N}^{*}} \subset L^{2}(0, T ; \mathbb{R})$ such that

$$
\int_{0}^{T} e^{-\lambda_{k, n} t} q_{j, n}(t) \mathrm{d} t=\delta_{k, j}, \quad \forall k, j \in \mathbb{N}^{*} .
$$

Thus, following the strategy proposed in [26, the control

$$
u_{n}(t, x)=-\sum_{k \in \mathbb{N}^{*}} e^{-\lambda_{k, n} T}\left\langle f_{n}^{0}, g_{k, n}\right\rangle_{L^{2}(-1,1)} \frac{g_{k, n}(x)}{\left\|g_{k, n}\right\|_{L^{2}(a, b)}^{2}} q_{k, n}(T-t),
$$

formally solves (1.5). It remains to prove that the above series actually converges and to get a bound on $u_{n}$ which is uniform with respect to $n$. Thus, the two main issues will be to give precise estimates (with respect to $k$ and $n$ ) of the following type

- a lower bound on $\left\|g_{k, n}\right\|_{L^{2}(a, b)}$;

- an upper bound on $\left\|q_{k, n}\right\|_{L^{2}(0, T)}$.

The first estimate is given in the following proposition.

Proposition 1.1. Let $a>0$. For any $\eta>0$ sufficiently small (depending only on a), there exists $C_{\eta}>0$ such that for any $k, n \in \mathbb{N}^{*}$,

$$
\int_{a}^{1} g_{k, n}^{2}(x) \mathrm{d} x \geq C_{\eta} \frac{e^{-n \pi\left(a^{2}+\eta\right)}}{n^{2} \pi^{2}} .
$$

This estimate is a straightforward application of [8, Proposition 4.5] (see for instance [8, Section 4.3]) and will not be detailed. The only difference is that the 
results in [8] give a lower bound on the $L^{2}((-1,-a) \cup(a, 1))$ norm. As the considered eigenfunctions are even or odd (see Lemma 2.1) this directly implies Proposition 1.1 . Note that (1.7) is an estimate of $g_{k, n}$ on $L^{2}(a, 1)$ and not $L^{2}(a, b)$. As mentioned in section 4.1 one can carry the analysis on the control domain $(a, 1) \times(0,1)$ then use a cut-off argument to prove the desired controllability property on $(a, b) \times(0,1)$.

The second estimate will require a careful spectral analysis of the operator $\mathcal{A}_{n}$. In this direction, let us recall the classical result obtained in [21.

Let $\rho>0$ and $\mathcal{N}: \mathbb{R}^{+} \rightarrow \mathbb{N}$. For an increasing sequence of positive numbers $\Theta=\left(\theta_{k}\right)_{k \geq 1}$ such that $\sum_{k} \frac{1}{\theta_{k}}<+\infty$ we say that $\Theta$ belongs to the class $\mathcal{L}(\rho, \mathcal{N})$ if

$$
\theta_{k+1}-\theta_{k}>\rho, \quad \forall k \in \mathbb{N}
$$

and

$$
\sum_{k \geq \mathcal{N}(\varepsilon)} \frac{1}{\theta_{k}} \leq \varepsilon, \quad \forall \varepsilon>0
$$

With such class of sequences H.O. Fattorini and D.L. Russell proved the following result (see [21, Theorem 1.5]).

Theorem 1.1. Let $\rho>0$ and $\mathcal{N}: \mathbb{R}^{+} \rightarrow \mathbb{N}$. For any $T>0$, for any $\varepsilon>0$, there exists a constant $C_{\varepsilon, T, \rho, \mathcal{N}}>0$ depending only on $\varepsilon, T, \rho$ and $\mathcal{N}$ such that, for any sequence $\Theta \in \mathcal{L}(\rho, \mathcal{N})$, there exists a biorthogonal family $\left(q_{\sigma}\right)_{\sigma \in \Theta}$ to the family of exponentials $\left(t \mapsto e^{-\theta t}\right)_{\theta \in \Theta}$ in $L^{2}(0, T ; \mathbb{R})$ satisfying

$$
\left\|q_{\sigma}\right\|_{L^{2}(0, T ; \mathbb{R})} \leq C_{\varepsilon, T, \rho, \mathcal{N}} e^{\varepsilon \sigma}, \quad \forall \sigma \in \Theta .
$$

In order to apply this result to estimate the control (1.6) achieving null controllability of (1.4), uniformly with respect to $n$, one should at least prove the following uniform (with respect to $n$ ) gap property

$$
\inf _{n \in \mathbb{N}^{*}}\left(\inf _{k \in \mathbb{N}^{*}}\left|\lambda_{k+1, n}-\lambda_{k, n}\right|\right)>0
$$

Even though numerical results seem to confirm that this property holds true (see Figure 1.2 , we were not able to prove it for the whole spectrum. Therefore, in order to conclude to the null controllability of (1.1) in optimal time, we will generalize the estimate of biorthogonal families given in Theorem 1.1 under weaker assumptions. This result is stated and discussed in Section 1.4.2 and may have its own interest in other situations.

The different spectral results obtained in the analysis of such property are presented in the next subsection.

Notation.

- In all this article, $C$ denotes a positive number that may vary from line to line. If necessary, we add information about dependency on parameters $p_{1}, p_{2}, \ldots, p_{k}$ in the following way: $C_{p_{1}, p_{2}, \ldots, p_{k}}$.

- For any $\alpha, \beta \in \mathbb{R}$, we will use the following notation: $\llbracket \alpha, \beta \rrbracket=\mathbb{N} \cap[\alpha, \beta]$.

- We shall use the Kronecker symbol $\delta_{x, y}$ which is 1 if $x=y$ and 0 otherwise.

- The real part (resp. imaginary part) of any complex number $z \in \mathbb{C}$ will be denoted by $\Re z$ (resp. $\Im z$ ). 


\subsection{Results.}

As already said in subsection 1.3. one of the main achievements concern the spectral analysis of operator $\mathcal{A}_{n}$. The results are presented in Section 1.4.1 and proved in Section 2 .

We also extend the results of [21] to obtain uniform estimates on biorthogonal families to real exponentials in the absence of a uniform gap property. This analysis, which has its own interest, is presented in Section 1.4 .2 and the detailed proofs are given in Section 3 .

Gathering these results, we finally obtain the null controllability of (1.1) for $T>$ $\frac{a^{2}}{2}$ using the moments method. This result is extended to cascade systems of Grushin equations. The Theorem is stated in Section 1.4.3 and proved in Section 4.

\subsubsection{Results on the spectrum of $\mathcal{A}_{n}$.}

To analyze the uniform gap property we have performed numerical simulations. In Figure 1.2 we represent, for different values of $n$, the gap $\lambda_{k+1, n}-\lambda_{k, n}$ divided by $n \pi$, as a function of $k$. It clearly appears that the uniform gap property 1.10 seems to hold true, and in fact even more since we observe that $\left|\lambda_{k+1, n}-\lambda_{k, n}\right| \geq 2 n \pi$ for any $k, n$. More precisely, this (normalized) gap appears to be roughly constant equal to 2 for low frequencies whereas it presents a linear growth in $k$ for high frequencies. Moreover, we observe that the position of the transition point between those two regimes seems to grow linearly with respect to the parameter $n$.

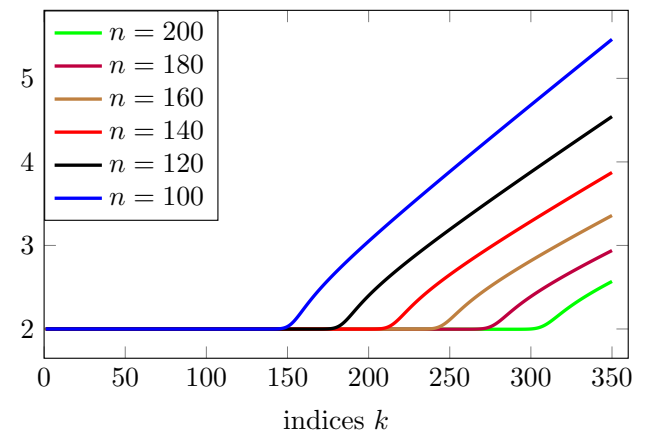

Figure 1.2: Gap divided by $n \pi: k \mapsto \frac{\lambda_{k+1, n}-\lambda_{k, n}}{n \pi}$.

To carefully analyze this gap property we will use two different strategies for low frequencies and high frequencies. Let us precisely define those two regimes: for any $\tau \in(0,1)$ and $n \in \mathbb{N}^{*}$, we define a lower and an upper range of indices by

$$
L_{\tau, n}:=\left\{k \in \mathbb{N}^{*}: k \leq \tau \frac{\pi}{2} n\right\} \quad \text { and } \quad U_{n}:=\left\{k \in \mathbb{N}^{*}: k \geq \frac{n \pi}{2}+1\right\} .
$$

The parameter $\tau$ is intended to be chosen close to 1 (see 4.5 in the controllability proof).

THEOREM 1.2. There exists $\gamma>0$ such that for any $\tau \in(0,1)$ there exists $n_{0}:=n_{0}(\tau) \in \mathbb{N}^{*}$ such that the following gap property holds

$$
\lambda_{k+1, n}-\lambda_{k, n}>\gamma, \quad \forall k \in L_{\tau, n} \cup U_{n}, \quad \forall n \geq n_{0} .
$$

Since for any $n \leq n_{0}(\tau)$ each sequence of eigenvalues $\left(\lambda_{k, n}\right)_{k \in \mathbb{N}^{*}}$ satisfies a gap property depending on $n$, the next result follows directly. 
Corollary 1.2. For any $\tau \in(0,1)$, there exists $\gamma_{\tau}>0$ such that, the following gap property holds

$$
\lambda_{k+1, n}-\lambda_{k, n}>\gamma_{\tau}, \quad \forall k \in L_{\tau, n} \cup U_{n}, \quad \forall n \geq 1 .
$$

As highlighted by Figure 1.2, the whole spectrum $\Lambda_{n}$ seems to enjoy a uniform gap property. However, we were not able to prove it for the indices in the intermediate set

$$
M_{\tau, n}:=\mathbb{N}^{*} \backslash\left(L_{\tau, n} \cup U_{n}\right),
$$

and obtained the much weaker estimate below, that will also play an important role in the analysis.

Proposition 1.2. Let $\tau \in\left[\frac{3}{4}, 1\right)$. For any $n \in \mathbb{N}^{*}$, we have

$$
\lambda_{k, n} \geq \frac{n^{2} \pi^{2}}{4}, \quad \forall k \geq \tau n \frac{\pi}{2}
$$

and

$$
\left|\lambda_{k, n}-\lambda_{j, n}\right| \geq \frac{\sqrt{\lambda_{k, n}}}{2} \exp \left(-\frac{8}{3} \sqrt{\lambda_{k, n}}\right), \quad \forall k \geq \tau n \frac{\pi}{2}, \forall j \neq k
$$

The last ingredient needed is the fact that, the number of eigenvalues in this intermediate region, even if it increases with $n$, can be chosen to be negligible in some sense since $\tau$ can be arbitrarily close to 1 . More precisely, we have

$$
\# M_{\tau, n} \leq(1-\tau) \frac{n \pi}{2}+1, \quad \forall \tau \in(0,1), \forall n \geq 1 .
$$

The key point to prove Theorem 1.1 (see Section 4 ) will be that there are not too much eigenvalues in the intermediate range: the factor $(1-\tau)$ will be crucial since we will be able to choose it as small as necessary.

\subsubsection{Existence and estimates of a biorthogonal family to the expo- nentials.}

As the uniform gap property stated in Theorem 1.2 is not established for the whole spectrum, one cannot apply Theorem 1.1 to obtain uniform estimates of the biorthogonal family to the associated exponentials. To cope with this difficulty, we extend the uniform estimates obtained in 21] under weaker assumptions.

Let us first introduce a few definitions. For any countable family $\Lambda \subset(0,+\infty)$, we denote by $N$ its counting function defined by

$$
N(r):=\#\{\lambda \in \Lambda ; \lambda \leq r\}, \quad \forall r \in \mathbb{R} .
$$

Moreover for any $\lambda \in \Lambda$ we define

$$
\rho_{\lambda}:=\operatorname{dist}(\lambda,\{0\} \cup \Lambda \backslash\{\lambda\}),
$$

and for any parameter $\gamma>0$, we set

$$
c_{\lambda}^{\gamma}:=\#((\Lambda \backslash\{\lambda\}) \cap(\lambda-\gamma, \lambda+\gamma)) .
$$


To simplify a little the notation, we do not explicitly mention that those three definitions depend on the whole family $\Lambda$.

Observe that

$$
\left(c_{\lambda}^{\gamma}=0, \quad \forall \lambda \in \Lambda\right) \Longleftrightarrow \inf _{\substack{\lambda, \mu \in \Lambda \\ \lambda \neq \mu}}|\lambda-\mu| \geq \gamma,
$$

so that $c_{\lambda}^{\gamma}$ can be interpreted as a local measure of the possible defect of a uniform gap with parameter $\gamma$ for the family $\Lambda$.

TheOREM 1.3. Let $T>0, \alpha \in(0,1)$ and $\bar{N}>0$. Let $\Lambda \subset(0,+\infty)$ be a countable family such that its counting function $N$ satisfies

$$
N(r) \leq \bar{N} r^{\alpha}, \quad \forall r>0 .
$$

Then, there exists a biorthogonal family $\left(q_{\lambda}\right)_{\lambda \in \Lambda}$ to the functions

$$
\left\{t \mapsto e^{-\lambda t} ; \lambda \in \Lambda\right\}
$$

in $L^{2}(0, T ; \mathbb{R})$ i.e.

$$
\int_{0}^{T} e^{-\lambda t} q_{\mu}(t) \mathrm{d} t=\delta_{\lambda, \mu}, \quad \forall \lambda, \mu \in \Lambda,
$$

such that for any $\lambda \in \Lambda$, the following estimate holds, for any $\gamma>0$,

$$
\left\|q_{\lambda}\right\|_{L^{2}(0, T)} \leq C_{T, \alpha, \bar{N}}\left(\frac{\lambda+\gamma}{\rho_{\lambda}}\right)^{c_{\lambda}^{\gamma}} \exp \left(C_{\alpha, \bar{N}}(1+\log (\lambda / \gamma)) \lambda^{\alpha}\right),
$$

where $C_{\alpha, \bar{N}}$ and $C_{T, \alpha, \bar{N}}$ only depend on the parameters $\alpha, \bar{N}$ (and $T$ ).

In Section 3 , this result is extended to biorthogonal families to

$$
\left\{t \mapsto(-t)^{p} e^{-\lambda t} ; p \in \llbracket 0, d \rrbracket, \lambda \in \Lambda\right\} .
$$

For more details we refer to Theorem 3.2 ,

The existence of a biorthogonal family as stated in Theorem 1.3 is classical (see for instance [28]). The main novelty is thus the estimate (1.21) which do not require any gap-type assumption and whose dependency with respect to the family $\Lambda$ is carefully given.

Let $\Lambda$ be a family satisfying the assumptions of Theorem 1.3 . Let us compare the estimate given in Theorem 1.3 with previously known results depending on the assumptions on $\Lambda$.

- If, in addition, $\Lambda$ satisfies the classical gap condition i.e. there exists $\gamma>0$ such that $|\lambda-\mu|>\gamma$ for any $\lambda, \mu \in \Lambda, \lambda \neq \mu$, then we have $c_{\lambda}^{\gamma}=0$ (see (1.19)). It follows that (1.21) reduces to

$$
\left\|q_{\lambda}\right\|_{L^{2}(0, T)} \leq C_{T, \alpha, \bar{N}} \exp \left(C_{\alpha, \bar{N}}(1+\log (\lambda / \gamma)) \lambda^{\alpha}\right) .
$$

This estimate is more accurate than the one given in [21. On the contrary, because of the $\log (\lambda)$ term in the exponential, this estimate is slightly suboptimal with respect to the one given in [9. In Remark 3.1. under a more accurate assumption on the asymptotic behaviour of $N$ than $[1.20)$, we drop this $\log$ term and thus recover the estimates of 9 .

For detailed estimates in the presence of a gap condition we refer to [15]. 
- If we assume now that $\Lambda$ satisfies a weak gap condition i.e. there exists $\gamma>0$ and $p \in \mathbb{N}^{*}$ such that

$$
\#(\Lambda \cap[\mu, \mu+\gamma]) \leq p, \quad \forall \mu>0,
$$

then we have $c_{\lambda}^{\gamma} \leq p-1$. With such weak gap condition, uniform estimates (with respect to different parameters) are given in [10] as a byproduct of the block moments method. We also mention the recent paper [23] where precise estimates, including time-dependency of the constants, are given in this setting.

Let us also mention that estimates for biorthogonal families with a weak gap condition (with $p=2$ ) were already obtained in [16]. In their work, they uses an extra assumption stating that the gap between two eigenvalues should not go to zero too quickly.

- In the absence of any (weak or strong) gap-type condition, the only previous result is 4 ] where the biorthogonal family is estimated using the condensation index.

In the case where the family $\Lambda$ satisfies a weak gap condition with $p=2$ i.e. there exists $\gamma>0$ such that

$$
\#(\Lambda \cap[\mu, \mu+\gamma]) \leq 2, \quad \forall \mu>0,
$$

we recover in 1.21) the same behavior when $\lambda \rightarrow+\infty$ as the one given in [4, 10, 23]. In more general situations, the estimate given in Theorem 1.3 is sub-optimal. However, with the spectral analysis of Theorem 1.2 , let us notice that the estimates given in [4, 10, 23] would not allow to prove Theorem 1.1. In fact, we will deal with the sequence of families $\Lambda_{n}$ which satisfies, for each $n$, a weak gap condition but with a parameter $p$ going to $+\infty$ with $n$.

Thus, it is crucial to notice that Theorem 1.3 do not require any gap-type condition and that the constants appearing in estimate (1.21) only depend on the asymptotic of the counting function.

To be completely accurate, let us precise that most of the above mentioned results apply in the slightly more general situation where the sequence $\Lambda$ is only assumed to satisfy $\sum_{\lambda \in \Lambda} \frac{1}{\lambda}<+\infty$, without any other assumption on the counting function. Our assumption (1.20) is a little bit more restrictive but the absence of any gap-type condition and the uniformity of the estimates offer a new variety of applications.

\subsubsection{Results on the minimal time of null controllability of (1.1).}

As already stated, one of the achievements of this article is to provide an alternative proof of Theorem 1.1 using the moments method. Let us roughly explain how the results presented in Sections 1.4.1 and 1.4.2 will eventually lead to Theorem 1.1.

As already mentioned, the new term in the estimate given in Theorem 1.3 is $\left(\frac{\lambda+\gamma}{\rho_{\lambda}}\right)^{c_{\lambda}^{\gamma}}$. In our application to Grushin equation (1.1), for eigenvalues $\lambda$ in the intermediate range where the uniform gap property is not proved, the size of this term will rely on the following two facts:

- from Proposition 1.2 the term $\rho_{\lambda}$ is of order $\exp (-\sqrt{\lambda})$;

- from (1.15) and (1.13), we have $c_{\lambda}^{\gamma}$ which is of order $(1-\tau) \sqrt{\lambda}$.

Thus, we will obtain a biorthogonal element of order $\exp ((1-\tau) \lambda+O(\sqrt{\lambda} \log \lambda))$. Choosing $\tau$ sufficiently close to 1 , the contribution of this biorthogonal element in the 
construction of the control (1.6) will be negligible compared to the dissipation. The need of a sufficiently large time will thus simply come from the insufficient observation of the eigenfunctions stated in Proposition 1.1. For more details, we refer to Section 4 and particularly estimate (4.4).

One of the main advantage of the machinery developed through the moments method is that Theorem 1.1 extends to cascade systems with one single control at no cost.

Let $d \geq 2$ and consider the controlled cascade system of $d$ Grushin equations

$$
\begin{cases}\partial_{t} F(t, x, y)+\left(\mathcal{D} I_{d}+K_{d}\right) F(t, x, y)=B_{d} 1_{\omega} u(t, x, y), & (t, x, y) \in(0, T) \times \Omega, \\ F(t, x, y)=0, & (t, x, y) \in(0, T) \times \partial \Omega, \\ F(0, x, y)=F^{0}(x, y) \in\left(L^{2}(\Omega)\right)^{d}, & (x, y) \in \Omega,\end{cases}
$$

where $F$ is now a vector of $\mathbb{R}^{d}$ and $I_{d}$ is the identity matrix of size $d$. The coupling matrix $K_{d}$ of size $d \times d$ and the control vector $B_{d} \in \mathbb{R}^{d}$ are defined as follows

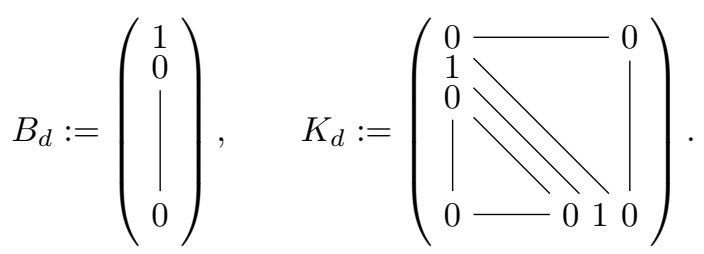

We then obtain the following result

TheOREm 1.4. Let $0<a<b \leq 1$. The minimal null control time of 1.22 with $\omega=(a, b) \times(0,1)$ is

$$
T^{*}=\frac{a^{2}}{2} .
$$

This theorem is proved in Section 4.2 . Notice that the first component of 1.22 is nothing but the controlled scalar Grushin equation (1.1). Thus, the lack of null controllability in time $T \leq \frac{a^{2}}{2}$ follows directly from $[6$.

Moreover, our strategy also allows to consider the following cascade system of $d$ Grushin equations with a single boundary control

$$
\begin{cases}\partial_{t} F(t, x, y)+\left(\mathcal{D} I_{d}+K_{d}\right) F(t, x, y)=0, & (t, x, y) \in(0, T) \times \Omega, \\ F(t, x, y)=0, & (t, x, y) \in(0, T) \times\left(\partial \Omega \backslash \Gamma_{1}\right), \\ F(t, x, y)=B_{d} u(t, y), & (t, x, y) \in(0, T) \times \Gamma_{1}, \\ F(0, x, y)=F^{0}(x, y) \in\left(L^{2}(\Omega)\right)^{d}, & (x, y) \in \Omega,\end{cases}
$$

where $\Gamma_{1}$ has been defined by $\Gamma_{1}=\{1\} \times[0,1]$. The functional space considered here for the boundary control is $L^{2}((0, T) \times(0,1))$. We then obtain the following result

TheOREM 1.5. The minimal null control time of 1.23 is

$$
T^{*}=\frac{1}{2} \text {. }
$$

This theorem is proved in Section 4.3. Contrarily to the scalar case, this result cannot be deduced from Theorem 1.4 using extensions and cut-off functions. It also seems to be out of reach using the Carleman inequalities as in 7 . 


\section{Spectral analysis of operator $\mathcal{A}_{n}$.}

In this section, we prove respectively Theorem 1.2 in $L_{\tau, n}$, Theorem 1.2 in $U_{n}$ and Proposition 1.2 .

\subsection{Study of the gap property.}

To deal with low frequencies it is fundamental to notice that the operator $\mathcal{A}_{n}$ is somehow related to the quantum harmonic oscillator. This idea was already present in [6]. Indeed, for any $n \in \mathbb{N}^{*}$, let $\Omega_{n}:=(-\sqrt{n \pi}, \sqrt{n \pi})$ and define the following operator

$$
\widetilde{\mathcal{A}}_{n}:=-\partial_{x x}+x^{2}, \quad D\left(\widetilde{\mathcal{A}}_{n}\right):=H^{2}\left(\Omega_{n}\right) \cap H_{0}^{1}\left(\Omega_{n}\right) .
$$

Introducing the following change of variables

$$
v_{k, n}(x):=\frac{1}{(n \pi)^{\frac{1}{4}}} g_{k, n}\left(\frac{x}{\sqrt{n \pi}}\right), \quad \forall x \in \Omega_{n}, \forall k \in \mathbb{N}^{*},
$$

it turns out that $v_{k, n}$ are eigenvectors of the truncated harmonic oscillator $\widetilde{\mathcal{A}}_{n}$ with associated eigenvalue $\frac{\lambda_{k, n}}{n \pi}$ i.e.

$$
\widetilde{\mathcal{A}}_{n} v_{k, n}=\frac{\lambda_{k, n}}{n \pi} v_{k, n} .
$$

Thus it is reasonable to expect, at least for large values of $n$, to obtain valuable informations from the quantum harmonic oscillator on the real line defined by

$$
\mathcal{A}:=-\partial_{x x}+x^{2}, \quad D(\mathcal{A}):=\left\{G \in H^{2}(\mathbb{R}) ; x \mapsto x G(x) \in L^{2}(\mathbb{R})\right\},
$$

and more precisely, on its eigenelements which are explicitly given by (see for instance [29])

$$
\mu_{k}=2 k-1, \quad G_{k}(x)=\frac{1}{\pi^{1 / 4} 2^{\frac{k-1}{2}} \sqrt{(k-1) !}} e^{-\frac{x^{2}}{2}} H_{k}(x), \quad \forall k \in \mathbb{N}^{*},
$$

where $H_{k}$ is the $(k-1)^{\text {th }}$ Hermite polynomial.

Let us notice that since the potential $x \mapsto x^{2}$ is even, the following Lemma holds.

Lemma 2.1. Let $n \in \mathbb{N}^{*}$.

- If $k$ is even, then $g_{k, n}, v_{k, n}$ and $G_{k}$ are odd functions.

- If $k$ is odd, then $g_{k, n}, v_{k, n}$ and $G_{k}$ are even functions.

The comparison between $\mathcal{A}$ and $\widetilde{\mathcal{A}}_{n}$ is at the heart of Section 2.1.2 where we prove the uniform gap-property for low frequencies. It will also allow us to obtain, in Section 2.1.1. a general lower bound on $\lambda_{k, n}$ that will be used all along this article. The regime of frequencies in which this comparison is relevant is exactly the one given by $L_{\tau, n}$ where, in some sense, $n$ is large with respect to $k$ (see Figure 1.2 ).

The gap property for high frequencies is proved in Section 2.1.3.

\subsubsection{A general lower bound on eigenvalues.}

We start proving an inequality, which is, unlike Theorem 1.2 , valid for the whole spectrum of $\mathcal{A}_{n}$.

Proposition 2.1. The following lower bound holds

$$
\lambda_{k, n} \geq n \pi(2 k-1), \quad \forall k, n \in \mathbb{N}^{*} .
$$


Proof. The proof is a direct consequence of the min-max principle. Let $k \in \mathbb{N}^{*}$ and consider the form domain of the operator $\mathcal{A}$ i.e.

$$
\mathcal{D}:=\left\{G \in H^{1}(\mathbb{R}) ; x \mapsto x G(x) \in L^{2}(\mathbb{R})\right\} .
$$

We have

$$
\mu_{k}=\min _{\substack{V_{k} \subset \mathcal{D} \\ \operatorname{dim} V_{k}=k}} \max _{G \in V_{k}} \frac{\int_{\mathbb{R}} G^{\prime}(x)^{2}+x^{2} G(x)^{2} \mathrm{~d} x}{\|G\|_{L^{2}(\mathbb{R})}^{2}} .
$$

For any $n \in \mathbb{N}^{*}$, let us consider

$$
V_{k, n}:=\operatorname{span}\left\{v_{1, n}, \ldots, v_{k, n}\right\},
$$

as a subspace of $L^{2}(\mathbb{R})$ where the functions $v_{j, n}$ are extended by 0 outside $\Omega_{n}$. Notice that,

$$
V_{k, n} \subset \mathcal{D}, \quad \text { and } \quad \operatorname{dim} V_{k, n}=k .
$$

Thus,

$\mu_{k} \leq \max _{\substack{G \in V_{k, n} \\\|G\|_{L^{2}(\mathbb{R})}=1}} \int_{\mathbb{R}} G^{\prime}(x)^{2}+x^{2} G(x)^{2} \mathrm{~d} x=\max _{\substack{G \in V_{k, n} \\\|G\|_{L^{2}\left(\Omega_{n}\right)}=1}} \int_{\Omega_{n}} G^{\prime}(x)^{2}+x^{2} G(x)^{2} \mathrm{~d} x=\frac{\lambda_{k, n}}{n \pi}$,

which ends the proof. $\square$

\subsubsection{Uniform gap property for low frequencies.}

We start dealing with low frequencies. As we will compare the truncated harmonic oscillator on $\Omega_{n}$ with the harmonic oscillator $\mathcal{A}$ we will need to prove that, if $k$ is not too large compared to $n$, the $k$-th eigenfunction $G_{k}$ of $\mathcal{A}$ is small outside the interval $\Omega_{n}=(-\sqrt{n \pi}, \sqrt{n \pi})$. We can precisely state the following two results, whose proofs are postponed to Appendix (see respectively Section 5.2 and Section 5.1).

LEMma 2.2. There exists an integer $n_{0} \in \mathbb{N}^{*}$ such that

$$
\int_{\Omega_{n}} G_{k}^{2}(x) \mathrm{d} x \geq \frac{1}{2}, \quad \forall k \leq \frac{\pi}{2} n, \quad \forall n \geq n_{0} .
$$

Lemma 2.3. Let $\tau \in(0,1)$. There exist $n_{0}(\tau) \in \mathbb{N}^{*}, C_{1}(\tau), C_{2}(\tau) \in \mathbb{R}_{+}^{*}$ such that

$$
0 \leq G_{k}(x) \leq C_{1} e^{-C_{2} n}, \quad \forall x \geq \sqrt{n \pi}, \quad \forall k \leq \tau n \frac{\pi}{2}, \quad \forall n \geq n_{0}
$$

The rest of this section is dedicated to the proof of the following proposition.

Proposition 2.2. Let $\tau \in(0,1)$. There exist $n_{0}(\tau) \in \mathbb{N}^{*}, c_{1}(\tau), c_{2}(\tau) \in \mathbb{R}_{+}^{*}$, such that

$$
\frac{\lambda_{k, n}}{n \pi} \leq \mu_{k}+c_{1} e^{-c_{2} n}, \quad \forall k \in L_{\tau, n}, \quad \forall n \geq n_{0} .
$$

Together with Proposition 2.1. this states that for low frequencies, the rescaled eigenvalues of the truncated harmonic oscillator are exponentially close (with respect 
to the parameter $n$ ) to the eigenvalues of the harmonic oscillator $\mathcal{A}$. As $\mu_{k}=2 k-1$, we immediately get the following corollary.

Corollary 2.1. Let $\tau \in(0,1)$. There exist $n_{0}(\tau) \in \mathbb{N}^{*}$ such that we have the gap property

$$
\lambda_{k+1, n}-\lambda_{k, n} \geq 1, \quad \forall k \in L_{\tau, n}, \quad \forall n \geq n_{0} .
$$

Proof (of Proposition 2.2). We fix $\tau \in(0,1)$. Let $n_{0}(\tau)$ be such that Lemmas 2.2 and 2.3 hold. We fix $n \geq n_{0}$ and we are going to prove 2.5 by a strong induction on $k$.

The case $k=1$ is already proved in [6, p. 83]. More precisely, those authors obtained

$$
\lambda_{1, n} \leq n \pi+O\left(n^{9 / 4} e^{-n \pi / 2}\right),
$$

which is indeed the desired bound. We will actually adapt the proof of [6] to get the general case.

Suppose now that the result is proved up to rank $k-1 \geq 1$.

- Case 1. $k$ is odd

In that case, $G_{k}$ is even. Let us define

$$
\widetilde{G}_{k}=\left(G_{k}-G_{k}(\sqrt{n \pi})\right)-\sum_{p=1}^{k-1} v_{p, n}\left(G_{k}-G_{k}(\sqrt{n \pi}), v_{p, n}\right)_{L^{2}\left(\Omega_{n}\right)} .
$$

Notice that, by construction, we have $\widetilde{G}_{k} \in \operatorname{span}\left(v_{1, n}, v_{2, n}, \ldots, v_{k-1, n}\right)^{\perp}$ and $\widetilde{G}_{k}( \pm \sqrt{n \pi})=0$. Thus, $\widetilde{G}_{k} \in H_{0}^{1}\left(\Omega_{n}\right)$ and from the max-min principle, it comes that

$$
\frac{\lambda_{k, n}}{n \pi} \leq \frac{\left(\widetilde{\mathcal{A}}_{n} \widetilde{G}_{k}, \widetilde{G}_{k}\right)_{L^{2}\left(\Omega_{n}\right)}}{\left\|\widetilde{G}_{k}\right\|_{L^{2}\left(\Omega_{n}\right)}^{2}}
$$

Let us now compute the image of $\widetilde{G}_{k}$ by the operator $\widetilde{\mathcal{A}}_{n}$.

$$
\begin{aligned}
\widetilde{\mathcal{A}}_{n} \widetilde{G}_{k}=-G_{k}^{\prime \prime}+x^{2} G_{k}-x^{2} G_{k}(\sqrt{n \pi}) \\
\quad-\sum_{p=1}^{k-1}\left(-v_{p, n}^{\prime \prime}+x^{2} v_{p, n}\right)\left(G_{k}-G_{k}(\sqrt{n \pi}), v_{p, n}\right)_{L^{2}\left(\Omega_{n}\right)} \\
=\mu_{k} G_{k}-x^{2} G_{k}(\sqrt{n \pi})-\sum_{p=1}^{k-1} \frac{\lambda_{p, n}}{n \pi} v_{p, n}\left(G_{k}-G_{k}(\sqrt{n \pi}), v_{p, n}\right)_{L^{2}\left(\Omega_{n}\right)} .
\end{aligned}
$$

Then, using that $\widetilde{G}_{k} \perp v_{p, n}$ for any $p \in\{1, \ldots, k-1\}$, and again the definition of $\widetilde{G}_{k}$, we find

$$
\begin{aligned}
\left(\mathcal{A} \widetilde{G}_{k}, \widetilde{G}_{k}\right)_{L^{2}\left(\Omega_{n}\right)} & =\int_{\Omega_{n}} \mu_{k} G_{k}(x) \widetilde{G}_{k}(x)-x^{2} G_{k}(\sqrt{n \pi}) \widetilde{G}_{k}(x) \mathrm{d} x \\
& =\int_{\Omega_{n}} \mu_{k} \widetilde{G}_{k}^{2}(x)+G_{k}(\sqrt{n \pi})\left(\mu_{k}-x^{2}\right) \widetilde{G}_{k}(x) \mathrm{d} x .
\end{aligned}
$$


This gives the following expression for the Rayleigh quotient we are interested in

$$
\frac{\left(\widetilde{\mathcal{A}}_{n} \widetilde{G}_{k}, \widetilde{G}_{k}\right)_{L^{2}\left(\Omega_{n}\right)}}{\left\|\widetilde{G}_{k}\right\|_{L^{2}\left(\Omega_{n}\right)}^{2}}=\mu_{k}+G_{k}(\sqrt{n \pi}) \frac{\int_{\Omega_{n}}\left(\mu_{k}-x^{2}\right) \widetilde{G}_{k}(x) \mathrm{d} x}{\left\|\widetilde{G}_{k}\right\|_{L^{2}\left(\Omega_{n}\right)}^{2}} .
$$

By 2.7) and the Cauchy-Schwarz inequality, we get

$$
\frac{\lambda_{k, n}}{n \pi} \leq \mu_{k}+G_{k}(\sqrt{n \pi}) \frac{\left\|\mu_{k}-x^{2}\right\|_{L^{2}\left(\Omega_{n}\right)}}{\left\|\widetilde{G}_{k}\right\|_{L^{2}\left(\Omega_{n}\right)}} .
$$

Note that $G_{k}(\sqrt{n \pi})$ is non negative as shown in Lemma 2.3 .

We now estimate the different terms in the previous right-hand side. The behavior of $G_{k}(\sqrt{n \pi})$ is given by Lemma 2.3 . Since $\mu_{k}=(2 k-1) \leq n \pi$, there exists $C^{\prime}>0$ independent of $n$ and $k$ (for instance, $C^{\prime}=\left(2+\frac{2}{5}\right) \pi^{5 / 2}$ ) such that:

$$
\left\|\mu_{k}-x^{2}\right\|_{L^{2}\left(\Omega_{n}\right)}^{2} \leq C^{\prime} n^{5 / 2} .
$$

Let us now estimate $\left\|\widetilde{G}_{k}\right\|_{L^{2}\left(\Omega_{n}\right)}$. Thanks to Parseval equality and the fact that $\widetilde{G}_{k} \in \operatorname{span}\left(v_{1, n}, v_{2, n}, \ldots, v_{k-1, n}\right)^{\perp}$, we have

$$
\int_{\Omega_{n}} \widetilde{G}_{k}^{2}(x) \mathrm{d} x=\sum_{p \geq 1}\left(\widetilde{G}_{k}, v_{p, n}\right)_{L^{2}\left(\Omega_{n}\right)}^{2}=\sum_{p \geq k}\left(\widetilde{G}_{k}, v_{p, n}\right)_{L^{2}\left(\Omega_{n}\right)}^{2} .
$$

Now, since $\left(v_{k, n}\right)_{k \geq 1}$ are pairwise orthogonal,

$$
\int_{\Omega_{n}} \widetilde{G}_{k}^{2}(x) \mathrm{d} x=\sum_{p \geq k}\left(G_{k}-G_{k}(\sqrt{n \pi}), v_{p, n}\right)_{L^{2}\left(\Omega_{n}\right)}^{2}
$$

and thus, using again Parseval equality,

$$
\int_{\Omega_{n}} \widetilde{G}_{k}^{2}(x) \mathrm{d} x=\int_{\Omega_{n}}\left(G_{k}(x)-G(\sqrt{n \pi})\right)^{2} \mathrm{~d} x-\sum_{p<k}\left(G_{k}-G_{k}(\sqrt{n \pi}), v_{p, n}\right)_{L^{2}\left(\Omega_{n}\right)}^{2} .
$$

Let us now look at $\left(G_{k}-G_{k}(\sqrt{n \pi}), v_{p, n}\right)_{L^{2}\left(\Omega_{n}\right)}^{2}$. We have

$$
\begin{aligned}
\left(G_{k}-G_{k}(\sqrt{n \pi}), v_{p, n}\right)_{L^{2}\left(\Omega_{n}\right)} \frac{\lambda_{p, n}}{n \pi}= & \left(G_{k}-G_{k}(\sqrt{n \pi}), \widetilde{\mathcal{A}}_{n} v_{p, n}\right)_{L^{2}\left(\Omega_{n}\right)} \\
= & \left(\widetilde{\mathcal{A}}_{n} G_{k}-x^{2} G_{k}(\sqrt{n \pi}), v_{p, n}\right)_{L^{2}\left(\Omega_{n}\right)} \\
= & \left(\mu_{k} G_{k}-x^{2} G_{k}(\sqrt{n \pi}), v_{p, n}\right)_{L^{2}\left(\Omega_{n}\right)} \\
= & \mu_{k}\left(G_{k}-G_{k}(\sqrt{n \pi}), v_{p, n}\right)_{L^{2}\left(\Omega_{n}\right)} \\
& +G_{k}(\sqrt{n \pi})\left(\mu_{k}-x^{2}, v_{p, n}\right)_{L^{2}\left(\Omega_{n}\right)} .
\end{aligned}
$$

Thus,

$$
\left(G_{k}-G_{k}(\sqrt{n \pi}), v_{p, n}\right)_{L^{2}\left(\Omega_{n}\right)}\left(\frac{\lambda_{p, n}}{n \pi}-\mu_{k}\right)=G_{k}(\sqrt{n \pi})\left(\mu_{k}-x^{2}, v_{p, n}\right)_{L^{2}\left(\Omega_{n}\right)}
$$


and,

$$
\left(G_{k}-G_{k}(\sqrt{n \pi}), v_{p, n}\right)_{L^{2}\left(\Omega_{n}\right)}^{2} \leq G_{k}(\sqrt{n \pi})^{2} \frac{\left\|\mu_{k}-x^{2}\right\|_{L^{2}\left(\Omega_{n}\right)}^{2}}{\left(\frac{\lambda_{p, n}}{n \pi}-\mu_{k}\right)^{2}}
$$

Let $p<k$. By the induction hypothesis,

$$
\frac{\lambda_{p, n}}{n \pi} \leq \mu_{p}+c_{1} e^{-c_{2} n} .
$$

As $c_{1}$ and $c_{2}$ only depend on $\tau$, we can increase $n_{0}$ (only depending on $\tau$ ) such that

$$
c_{1} e^{-c_{2} n} \leq 1, \quad \forall n \geq n_{0}(\tau) .
$$

Therefore,

$$
\mu_{k}-\frac{\lambda_{p, n}}{n \pi} \geq \mu_{k}-\left(\mu_{p}+1\right) \geq 2(k-p)-1>0 .
$$

Thus, with 2.13 , we obtain

$$
\sum_{p<k}\left(G_{k}-G_{k}(\sqrt{n \pi}), v_{p, n}\right)_{L^{2}\left(\Omega_{n}\right)}^{2} \leq \frac{\pi^{2}}{6} G_{k}(\sqrt{n \pi})^{2}\left\|\mu_{k}-x^{2}\right\|_{L^{2}\left(\Omega_{n}\right)}^{2} .
$$

Using 2.10 and plugging it in 2.12 it comes that

$$
\int_{\Omega_{n}} \widetilde{G}_{k}^{2}(x) \mathrm{d} x \geq \int_{\Omega_{n}}\left(G_{k}(x)-G_{k}(\sqrt{n \pi})\right)^{2} \mathrm{~d} x-\frac{\pi^{2}}{6} C^{\prime} n^{5 / 2} G_{k}(\sqrt{n \pi})^{2} .
$$

We now estimate $\int_{\Omega_{n}}\left(G_{k}(x)-G_{k}(\sqrt{n \pi})\right)^{2} \mathrm{~d} x$ thanks to Lemma 2.2 Indeed, as $n \geq n_{0}$, we have

$$
\begin{aligned}
\int_{\Omega_{n}}\left(G_{k}(x)-G_{k}(\sqrt{n \pi})\right)^{2} \mathrm{~d} x & \geq \frac{1}{2} \int_{\Omega_{n}} G_{k}^{2}(x) \mathrm{d} x-2 \sqrt{n \pi} G_{k}^{2}(\sqrt{n \pi}) \\
& \geq \frac{1}{4}-2 \sqrt{n \pi} G_{k}^{2}(\sqrt{n \pi}) .
\end{aligned}
$$

Going back to 2.16), we obtain

$$
\int_{\Omega_{n}} \widetilde{G}_{k}^{2}(x) \mathrm{d} x \geq \frac{1}{4}-G_{k}(\sqrt{n \pi})^{2}\left(2 \sqrt{n \pi}+\frac{\pi^{2}}{6} C^{\prime} n^{5 / 2}\right) .
$$

Finally, using (2.4), for $n$ sufficiently large, depending only on $\tau$, we obtain

$$
\int_{\Omega_{n}} \widetilde{G}_{k}^{2}(x) \mathrm{d} x \geq \frac{1}{8} .
$$

Then, using 2.10, 2.18 in 2.9 leads to

$$
\frac{\lambda_{k, n}}{n \pi} \leq \mu_{k}+\sqrt{8 C^{\prime}} n^{5 / 4} G_{k}(\sqrt{n \pi})
$$

The estimate of Lemma 2.3 proves 2.5 when $k$ is odd. 
- Case 2. $k$ is even.

In that case, $G_{k}$ is odd and we set now:

$$
\widetilde{G}_{k}=\left(G_{k}-\frac{x}{\sqrt{n \pi}} G_{k}(\sqrt{n \pi})\right)-\sum_{p=1}^{k-1} v_{p, n}\left(G_{k}-\frac{x}{\sqrt{n \pi}} G_{k}(\sqrt{n \pi}), v_{p, n}\right)_{L^{2}\left(\Omega_{n}\right)} .
$$

Inequality 2.8 becomes:

$$
\frac{\int_{\Omega_{n}}\left(\widetilde{G}_{k}^{\prime}(x)\right)^{2}+x^{2} \widetilde{G}_{k}^{2}(x) \mathrm{d} x}{\left\|\widetilde{G}_{k}\right\|_{L^{2}\left(\Omega_{n}\right)}^{2}}=\mu_{k}+G_{k}(\sqrt{n \pi}) \frac{\int_{\Omega_{n}}\left(\mu_{k}-\frac{x^{3}}{\sqrt{n \pi}}\right) \widetilde{G}_{k}(x) \mathrm{d} x}{\left\|\widetilde{G}_{k}\right\|_{L^{2}\left(\Omega_{n}\right)}^{2}} .
$$

Inequality 2.10 is replaced by

$$
\left\|\mu_{k}-\frac{x^{3}}{\sqrt{n \pi}}\right\|_{L^{2}\left(\Omega_{n}\right)}^{2} \leq C^{\prime} n^{5 / 2}
$$

with $C^{\prime}=\left(2+\frac{2}{7}\right) \pi^{5 / 2}$ and we conclude in the exact same way.

\subsubsection{Uniform gap property for high frequencies.}

The goal of this section is to prove the following theorem:

THEOREM 2.1. We have the lower bound

$$
\lambda_{k+1, n}-\lambda_{k, n} \geq \frac{\pi}{1+\pi},
$$

for any $k, n \geq 1$ for which $\lambda_{k, n}$ satisfies

$$
\lambda_{k, n} \geq n^{2} \pi^{2}+n \pi
$$

Remark 2.1. According to Proposition 2.1, condition (2.21) is satisfied in particular when $k \in U_{n}$.

Proof. Let $k, n \in \mathbb{N}^{*}$, let us recall the spectral problem satisfied by the couple $\left(\lambda_{k, n}, g_{k, n}\right)$

$$
\left\{\begin{array}{l}
-\partial_{x x} g_{k, n}(x)+(n \pi x)^{2} g_{k, n}(x)=\lambda_{k, n} g_{k, n}(x), \text { for } x \in(-1,1), \\
g_{k, n}( \pm 1)=0
\end{array}\right.
$$

and we assume, possibly changing the sign of $g_{k, n}$, that $\partial_{x} g_{k, n}(-1)>0$.

We use the idea of [27, where the authors replace the eigenvalue $\lambda_{k, n}$ by a new variable, denoted here by $\lambda$. Hence, for any $\lambda \in \mathbb{R}$, we consider the following Cauchy problem:

$$
\left\{\begin{array}{l}
-\partial_{x x} h_{n}(\lambda, x)+(n \pi x)^{2} h_{n}(\lambda, x)=\lambda h_{n}(\lambda, x), \text { for } x \in(-1,1) \\
h_{n}(\lambda,-1)=0 \\
\partial_{x} h_{n}(\lambda,-1) \text { is positive and chosen so that }\left\|h_{n}(\lambda, \bullet)\right\|_{L^{2}(-1,1)}=1
\end{array}\right.
$$

The modified Prüfer's change of variable consists in introducing two functions $\rho_{n}>0$ and $\theta_{n}$ satisfying :

$$
\left\{\begin{array}{l}
h_{n}(\lambda, x)=\rho_{n}(\lambda, x) \cos \left(\theta_{n}(\lambda, x)\right)\left(\lambda-n^{2} \pi^{2} x^{2}\right)^{-1 / 4} \\
\partial_{x} h_{n}(\lambda, x)=\rho_{n}(\lambda, x) \sin \left(\theta_{n}(\lambda, x)\right)\left(\lambda-n^{2} \pi^{2} x^{2}\right)^{1 / 4}
\end{array}\right.
$$


We impose that the variable $\lambda$ satisfies $\lambda>n^{2} \pi^{2}$, so that the expressions in 2.24 make sense. Under this condition, existence of $\theta_{n}$ and $\rho_{n}$ is a classical result. Notice that, since $h_{n}(\lambda,-1)=0$ and $\partial_{x} h_{n}(\lambda,-1)$ is positive, we can impose without loss of generality

$$
\theta_{n}(\lambda,-1)=\frac{\pi}{2}
$$

and in that case $\theta_{n}$ and $\rho_{n}$ are uniquely determined.

Moreover, one can show (see [11] or 24]) that $\theta_{n}$ is the unique solution of the following Cauchy problem

$$
\left\{\begin{array}{l}
\partial_{x} \theta_{n}(\lambda, x)=-\sqrt{\lambda-n^{2} \pi^{2} x^{2}}+\frac{n^{2} \pi^{2} x \sin \left(2 \theta_{n}(\lambda, x)\right)}{2\left(\lambda-n^{2} \pi^{2} x^{2}\right)}, \text { for } x \in[-1,1], \\
\theta_{n}(\lambda,-1)=\frac{\pi}{2}
\end{array}\right.
$$
2].

Let us start with the following lemma which is given without proof in 22, Lemma

Lemma 2.4. Let $\lambda_{k, n}$ be an eigenvalue such that $\lambda_{k, n} \geq n^{2} \pi^{2}+n \pi$. The solution $\theta_{n}$ of 2.25 satisfies

$$
\theta_{n}\left(\lambda_{k, n}, 1\right)=\frac{\pi}{2}-k \pi
$$

Proof. First, notice that $h_{n}\left(\lambda_{k, n}, x\right)=g_{k, n}(x)$. According to Sturm oscillation Theorem, the function $x \in[-1,1] \mapsto h_{n}\left(\lambda_{k, n}, x\right)$ has exactly $k+1$ zeros. Thus, given that $\rho_{n}$ does not vanishes in $[-1,1]$, there exists exactly $k+1$ points $-1=x_{0}<x_{1}<$ $\cdots<x_{k}=1$ such that $\forall i \in \llbracket 0, k \rrbracket, \theta_{n}\left(\lambda_{k, n}, x_{i}\right)=\pi / 2 \bmod \pi$.

Since $\theta_{n}\left(\lambda_{k, n}, x_{0}\right)=\frac{\pi}{2}$, by continuity of $x \mapsto \theta_{n}\left(\lambda_{k, n}, x\right)$ we have $\theta_{n}\left(\lambda_{k, n}, x_{1}\right) \in$ $\left\{\frac{\pi}{2}-\pi, \frac{\pi}{2}, \frac{\pi}{2}+\pi\right\}$. But according to 2.25), $\partial_{x} \theta_{n}\left(\lambda_{k, n}, x_{0}\right)=-\sqrt{\lambda-n^{2} \pi^{2} x_{0}^{2}}<0$ thus $\theta_{n}\left(\lambda_{k, n}, x_{1}\right) \neq \frac{\pi}{2}+\pi$, otherwise, by continuity of $x \mapsto \theta_{n}\left(\lambda_{k, n}, x\right)$ there would exist a point $x \in\left(x_{0}, x_{1}\right)$ such that $\theta_{n}\left(\lambda_{k, n}, x\right)=\pi / 2$. Since also $\partial_{x} \theta_{n}\left(\lambda_{k, n}, x_{1}\right)<0$, $\theta_{n}\left(\lambda_{k, n}, x_{1}\right) \neq \frac{\pi}{2}$, hence $\theta_{n}\left(\lambda_{k, n}, x_{1}\right)=\frac{\pi}{2}-\pi$.

We deduce with an immediate induction argument that $\theta_{n}\left(\lambda_{k, n}, x_{k}\right)=\frac{\pi}{2}-k \pi$.

According to Lemma 2.4 and the fact that $\lambda \mapsto \theta_{n}(\lambda, 1)$ is continously differentiable (see [30, Theorem 2.5.2]), we have

$$
\theta_{n}\left(\lambda_{k+1, n}, 1\right)-\theta_{n}\left(\lambda_{k, n}, 1\right)=-\pi=\int_{\lambda_{k, n}}^{\lambda_{k+1, n}} \partial_{\lambda} \theta_{n}(\lambda, 1) \mathrm{d} \lambda
$$

It follows that

$$
\pi \leq\left(\lambda_{k+1, n}-\lambda_{k, n}\right) \sup _{\lambda \in\left[\lambda_{k, n}, \lambda_{k+1, n}\right]}\left|\partial_{\lambda} \theta_{n}(\lambda, 1)\right|,
$$

We shall now estimate this supremum. For this purpose, let's compute the derivative with respect to $\lambda$ of 2.25 :

$$
\partial_{\lambda}\left(\partial_{x} \theta_{n}\right)=-\frac{1}{2 \sqrt{\lambda-n^{2} \pi^{2} x^{2}}}-\frac{n^{2} \pi^{2} x}{2\left(\lambda-n^{2} \pi^{2} x^{2}\right)^{2}} \sin \left(2 \theta_{n}\right)+\frac{n^{2} \pi^{2} x \cos \left(2 \theta_{n}\right)}{\lambda-n^{2} \pi^{2} x^{2}} \partial_{\lambda} \theta_{n}
$$


by regularity of $\theta_{n}$, we can exchange the derivatives and use that $\partial_{\lambda} \theta_{n}(\lambda,-1)=0$ to get, for any $x \in(-1,1)$,

$$
\begin{aligned}
\partial_{\lambda} \theta_{n}(\lambda, x)=\int_{-1}^{x}\left(-\frac{1}{2 \sqrt{\lambda-n^{2} \pi^{2} y^{2}}}-\frac{n^{2} \pi^{2} y}{2\left(\lambda-n^{2} \pi^{2} y^{2}\right)^{2}} \sin \left(2 \theta_{n}(\lambda, y)\right)\right. \\
\left.+\frac{n^{2} \pi^{2} y \cos \left(2 \theta_{n}(\lambda, y)\right)}{\lambda-n^{2} \pi^{2} y^{2}} \partial_{\lambda} \theta_{n}(\lambda, y)\right) \mathrm{d} y,
\end{aligned}
$$

so that

$$
\left|\partial_{\lambda} \theta_{n}(\lambda, x)\right| \leq \int_{-1}^{x}\left(\frac{1}{2 \sqrt{\lambda-n^{2} \pi^{2} y^{2}}}+\frac{n^{2} \pi^{2}|y|}{2\left(\lambda-n^{2} \pi^{2} y^{2}\right)^{2}}+\frac{n^{2} \pi^{2}|y|}{\lambda-n^{2} \pi^{2} y^{2}}\left|\partial_{\lambda} \theta_{n}(\lambda, y)\right|\right) \mathrm{d} y .
$$

Applying Gronwall's Lemma, we obtain

$$
\begin{aligned}
\left|\partial_{\lambda} \theta_{n}(\lambda, 1)\right| \leq & \int_{-1}^{1} \frac{1}{2 \sqrt{\lambda-n^{2} \pi^{2} y^{2}}} \exp \left(\int_{y}^{1} \frac{n^{2} \pi^{2}|s|}{\lambda-n^{2} \pi^{2} s^{2}} \mathrm{~d} s\right) \mathrm{d} y \\
& +\int_{-1}^{1} \frac{n^{2} \pi^{2}|y|}{2\left(\lambda-n^{2} \pi^{2} y^{2}\right)^{2}} \exp \left(\int_{y}^{1} \frac{n^{2} \pi^{2}|s|}{\lambda-n^{2} \pi^{2} s^{2}} \mathrm{~d} s\right) \mathrm{d} y,
\end{aligned}
$$

and finally, using the fact that the functions involved are even,

$$
\begin{aligned}
\left|\partial_{\lambda} \theta_{n}(\lambda, 1)\right| \leq & \left(\int_{0}^{1} \frac{1}{\sqrt{\lambda-n^{2} \pi^{2} y^{2}}} \mathrm{~d} y+\int_{0}^{1} \frac{n^{2} \pi^{2} y}{\left(\lambda-n^{2} \pi^{2} y^{2}\right)^{2}} \mathrm{~d} y\right) \\
& \times \exp \left(\int_{0}^{1} \frac{2 n^{2} \pi^{2} s}{\lambda-n^{2} \pi^{2} s^{2}} \mathrm{~d} s\right) .
\end{aligned}
$$

The three integrals appearing in the right-hand side can be bounded explicitely (we recall that $\left.\lambda>n^{2} \pi^{2}+n \pi\right)$ as follows

$$
\begin{gathered}
\int_{0}^{1} \frac{1}{\sqrt{\lambda-n^{2} \pi^{2} y^{2}}} \mathrm{~d} y=\frac{1}{n \pi} \int_{0}^{\frac{n \pi}{\sqrt{\lambda}}} \frac{d t}{\sqrt{1-t^{2}}} \leq \frac{1}{2 n}, \\
\int_{0}^{1} \frac{n^{2} \pi^{2} y}{\left(\lambda-n^{2} \pi^{2} y^{2}\right)^{2}} \mathrm{~d} y=\frac{1}{2}\left[\frac{1}{\left(\lambda-n^{2} \pi^{2} y^{2}\right)}\right]_{0}^{1} \leq \frac{1}{2\left(\lambda-n^{2} \pi^{2}\right)} \leq \frac{1}{2 n \pi},
\end{gathered}
$$

and

$$
\int_{0}^{1} \frac{2 n^{2} \pi^{2} s}{\lambda-n^{2} \pi^{2} s^{2}} \mathrm{~d} s=\left[-\log \left(\lambda-n^{2} \pi^{2} s^{2}\right)\right]_{0}^{1}=\log \left(\frac{\lambda}{\lambda-n^{2} \pi^{2}}\right) \leq \log (1+n \pi) .
$$

Gathering those estimates in 2.28, we finally get

$$
\left|\partial_{\lambda} \theta_{n}(\lambda, 1)\right| \leq\left(\frac{1}{2 n}+\frac{1}{n \pi}\right) e^{\log (1+n \pi)} \leq \frac{1+n \pi}{n} \leq 1+\pi .
$$

Injecting this upper bound in 2.26 , we get

$$
\pi \leq\left(\lambda_{k+1, n}-\lambda_{k, n}\right)(1+\pi),
$$


and the claim is proved.

REMARK 2.2. In [1, Theorem 4.5.1], it is proved that for any $\varepsilon>\frac{1}{\exp (\pi)-1}>0$, there exist $C_{\varepsilon}>0$ and $n_{0} \in \mathbb{N}^{*}$ both depending only on $\varepsilon$ such that

$$
\lambda_{k+1, n}-\lambda_{k, n} \geq C_{\varepsilon} \sqrt{\lambda_{k, n}}, \quad \forall n \geq n_{0}, \forall k \geq \frac{\pi}{2} n(1+\varepsilon) .
$$

The range of application of this estimate is slightly more restrictive than $U_{n}$ but gives a lower bound for the gap that goes to infinity with $k$. This is coherent with the linear behavior observed on the gap in Figure 1.2 for high frequencies and with the general theory of Sturm Liouville operators stating that, for a fixed $n \in \mathbb{N}^{*}$, one recovers an asymptotic quadratic behavior for the eigenvalues (see for instance [2, Theorem 1.1]).

\subsubsection{Conclusion.}

We have proved two gap properties, one for the eigenvalues whose indices lie in $L_{\tau, n}$ and another one for the eigenvalues whose indices belong to $U_{n}$. To conclude, let us show that the gap property holds for the union: $L_{\tau, n} \cup U_{n}$. Take $k_{1} \in L_{\tau, n}$ and $k_{2} \in U_{n}$. According to Proposition 2.2 and estimate 2.14,

$$
\frac{\lambda_{k_{1}, n}}{n \pi} \leq\left(2 k_{1}-1\right)+1=2 k_{1} .
$$

Hence, applying Proposition 2.1 with $k_{2} \in U_{n}$,

$$
\lambda_{k_{2}, n}-\lambda_{k_{1}, n} \geq n \pi\left(2\left(k_{2}-k_{1}\right)-1\right) \geq n \pi .
$$

\subsection{Non-uniform gap estimate for middle frequencies.}

This section is dedicated to the proof of Proposition 1.2

From Proposition 2.1, as $\tau \geq \frac{3}{4}$, we have

$$
k \geq \tau n \frac{\pi}{2} \quad \Longrightarrow \quad \lambda_{k, n} \geq n \pi\left(2 \tau n \frac{\pi}{2}-1\right) \geq \frac{3}{4} n^{2} \pi^{2}-n \pi,
$$

which implies (1.13).

The estimate (1.14) will follow adapting the arguments of [2, Theorem 1.1]. Let $\lambda$ and $\tilde{\lambda}$ in $\Lambda_{n}$. Let $u$ and $\tilde{u}$ the associated normalized eigenfunctions. Setting

$$
w(x):=u^{\prime}(-1) \tilde{u}(x)-\tilde{u}^{\prime}(-1) u(x),
$$

we obtain $w(-1)=w^{\prime}(-1)=0$ and

$$
-w^{\prime \prime}(x)+n^{2} \pi^{2} x^{2} w(x)=\lambda w+(\tilde{\lambda}-\lambda) u^{\prime}(-1) \tilde{u}(x) .
$$

Following [2, Lemma 2.2 and proof of Lemma 2.3], if we set $W=\left(\begin{array}{c}w \\ \frac{w^{\prime}}{\sqrt{\lambda}}\end{array}\right)$ we obtain

$$
\begin{aligned}
\|W(x)\| & \leq \exp \left(\int_{-1}^{x} \frac{n^{2} \pi^{2} s^{2}}{\sqrt{\lambda}} \mathrm{d} s\right) \int_{-1}^{x}\left|\frac{\widetilde{\lambda}-\lambda}{\sqrt{\lambda}} u^{\prime}(-1) \tilde{u}(s)\right| \mathrm{d} s \\
& \leq \sqrt{2} \exp \left(\frac{2}{3} \frac{n^{2} \pi^{2}}{\sqrt{\lambda}}\right) \frac{|\widetilde{\lambda}-\lambda|}{\sqrt{\lambda}}\left|u^{\prime}(-1)\right| .
\end{aligned}
$$


Noticing that the two eigenfunctions are normalized and orthogonal, thus

$$
\begin{aligned}
\left|u^{\prime}(-1)\right|^{2} & \leq \int_{-1}^{1}\left|u^{\prime}(-1) \tilde{u}(s)-\tilde{u}^{\prime}(-1) u(s)\right|^{2} \mathrm{~d} s \leq \int_{-1}^{1}\|W(s)\|^{2} \mathrm{~d} s \\
& \leq 2\left(\sqrt{2} \exp \left(\frac{2}{3} \frac{n^{2} \pi^{2}}{\sqrt{\lambda}}\right) \frac{|\widetilde{\lambda}-\lambda|}{\sqrt{\lambda}}\right)^{2}\left|u^{\prime}(-1)\right|^{2}
\end{aligned}
$$

As $u^{\prime}(-1) \neq 0$, it comes that

$$
|\lambda-\widetilde{\lambda}| \geq \frac{\sqrt{\lambda}}{2} \exp \left(-\frac{2}{3} \frac{n^{2} \pi^{2}}{\sqrt{\lambda}}\right) .
$$

Let $k \geq \tau n \frac{\pi}{2}$ and $j \geq 1, j \neq k$. We apply this estimate to $\lambda=\lambda_{k, n}$ and $\widetilde{\lambda}=\lambda_{j, n}$. Taking into account (1.13), we obtain

$$
\left|\lambda_{k, n}-\lambda_{j, n}\right| \geq \frac{\sqrt{\lambda_{k, n}}}{2} \exp \left(-\frac{2}{3} \frac{4 \lambda_{k, n}}{\sqrt{\lambda_{k, n}}}\right) .
$$

which ends the proof of Proposition 1.2 .

\section{The biorthogonal family.}

The goal of this section is to prove the existence of a biorthogonal family to the exponentials as well as the suitable estimates stated in Theorem 1.3. We start with some technical estimates on the inverse of Blaschke-type products and their derivatives in the considered setting. We use these estimates in Section 3.2 to prove Theorem 1.3 and finally extend it to biorthogonal families to

$$
\left\{t \mapsto(-t)^{p} e^{-\lambda t} ; p \in \llbracket 0, d \rrbracket, \lambda \in \Lambda\right\},
$$

in Theorem 3.2 ,

\subsection{Preliminary estimates.}

We use in this section the notation introduced in Section 1.4.2, in particular (1.16), 1.17) and 1.18.

For any countable family $\Lambda \subset(0,+\infty)$ such that $\sum_{\lambda \in \Lambda} \frac{1}{\lambda}<+\infty$ and any $\lambda \in \Lambda$, we define the Blaschke product

$$
W_{\lambda}^{\Lambda}(z):=\prod_{\substack{\sigma \in \Lambda \\ \sigma \neq \lambda}} \frac{\sigma-z}{\sigma+z}, \quad \forall z \in \mathbb{C},
$$

which is well-defined and holomorphic on $\mathbb{C}$ due to the summability assumption on $\left(\frac{1}{\lambda}\right)_{\lambda \in \Lambda}$.

\subsubsection{Estimates of Blaschke product.}

We start with the following estimate of the reciprocal of the Blaschke product in the neighborhood of $\lambda$.

Proposition 3.1. Let $\alpha \in(0,1)$ and $\bar{N}>0$. 
- Let $\Lambda$ be a family such that its counting function $N$ satisfies 1.20 .

For any $\lambda \in \Lambda$, we have the estimate

$$
\begin{aligned}
\left|\frac{1}{W_{\lambda}^{\Lambda}(x)}\right| \leq & \left(\frac{x+2 \gamma}{\rho_{\lambda}}\right)^{c_{\lambda}^{\gamma}} \exp \left(C_{\alpha, \bar{N}}(1+\log (x / \gamma)) x^{\alpha}\right), \\
& \forall \gamma>0, \quad \forall x \in \mathbb{R} \text {, s.t. }|x-\lambda| \leq \frac{\min \left(\gamma, \rho_{\lambda}\right)}{2},
\end{aligned}
$$

where $C_{\alpha, \bar{N}}$ only depends on the parameters $\alpha$ and $\bar{N}$.

- Let $\Lambda$ be a family such that its counting function $N$ satisfies the more precise asymptotic

$$
\left|N(r)-\bar{N} r^{\alpha}\right| \leq C, \quad \forall r>0 .
$$

For any $\lambda \in \Lambda$, we have the estimate

$$
\begin{aligned}
&\left|\frac{1}{W_{\lambda}^{\Lambda}(x)}\right| \leq\left(\frac{x+2 \gamma}{\rho_{\lambda}}\right)^{c_{\lambda}^{\gamma}} \exp \left(C_{\alpha, \bar{N}}(1-\log (\gamma)) x^{\alpha}\right), \\
& \forall \gamma>0, \quad \forall x \in \mathbb{R}, \text { s.t. }|x-\lambda| \leq \frac{\min \left(\gamma, \rho_{\lambda}\right)}{2},
\end{aligned}
$$

where $C_{\alpha, \bar{N}}$ only depends on the parameters $\alpha$ and $\bar{N}$.

REMARK 3.1. The difference between the two estimates given in the previous proposition is that, under the stronger assumption (3.2), there is no $\log x$ term in the exponential.

Thanks to this slight improvement, the present strategy developed in the rest of the article let us exactly recover the estimates of biorthogonal families under a gap assumption as given in [9, Theorem 1.5] with constants depending only on $\alpha$ and $\bar{N}$ and on the spectral gap $\rho$.

However, for our purpose, we focus on the weaker assumption $(1.20)$. Thus, in the following sections we will not consider both settings.

Before proving Proposition 3.1, let us recall some useful formulas making use of the counting function. These results are straightforward and we omit the proofs. For detailed proofs we refer to [14].

Lemma 3.1. Let $f:(0,+\infty) \rightarrow \mathbb{R}$ be of class $\mathcal{C}^{1}$.

- For any $r>0$,

$$
\sum_{\substack{\lambda \in \Lambda \\ \lambda \leq r}} f(\lambda)=f(r) N(r)-\int_{0}^{r} f^{\prime}(t) N(t) \mathrm{d} t .
$$

- For any $0 \leq s<r$,

$$
\sum_{\substack{\lambda \in \Lambda \\ s<\lambda \leq r}} f(\lambda)=f(r) N(r)-f(s) N(s)-\int_{s}^{r} f^{\prime}(t) N(t) \mathrm{d} t .
$$

- For any $r>0$ such that the following quantities are well defined,

$$
\sum_{\substack{\lambda \in \Lambda \\ \lambda>r}} f(\lambda)=-f(r) N(r)-\int_{r}^{+\infty} f^{\prime}(t) N(t) \mathrm{d} t .
$$


From now on, we introduce the remainder of the series of the reciprocals of the eigenvalues

$$
R(r):=\sum_{\substack{\lambda \in \Lambda \\ \lambda>r}} \frac{1}{\lambda}
$$

and we observe that it is related to the counting function through the formula

$$
N(r)-N(s) \leq r R(s), \quad \forall 0<s<r .
$$

Lemma 3.2. Assume that the counting function associated with a family $\Lambda$ satisfies 1.20 then we have

$$
\inf \Lambda \geq\left(\frac{1}{\bar{N}}\right)^{\frac{1}{\alpha}}
$$

and

$$
R(r) \leq \frac{\bar{N}}{1-\alpha} r^{\alpha-1}, \quad \forall r>0
$$

We now have all the ingredients to proceed to the proof of Proposition 3.1 .

Proof (of Proposition 3.1).

We first observe that, by definition of $\rho_{\lambda}$ we have

$$
\left(\lambda-\rho_{\lambda}, \lambda+\rho_{\lambda}\right) \cap \Lambda=\{\lambda\}
$$

and in particular, since $|x-\lambda| \leq \rho_{\lambda} / 2$, we have

$$
(0, x) \cap \Lambda=(0, \lambda) \cap \Lambda, \text { and }(x,+\infty) \cap \Lambda=(\lambda,+\infty) \cap \Lambda .
$$

We will prove the estimate under the assumption that

$$
x+\frac{\gamma}{2} \notin \Lambda,
$$

the general case being deduced by density.

Let us write the Blaschke product as

$$
\left|\frac{1}{W_{\lambda}^{\Lambda}(x)}\right|=\frac{\prod_{\substack{\sigma \in \Lambda \\ \sigma \neq \lambda}}\left(1+\frac{x}{\sigma}\right)}{\prod_{\substack{\sigma \in \Lambda \\ \sigma \neq \lambda}}\left|1-\frac{x}{\sigma}\right|}=: \frac{Q(x)}{D(x)} .
$$

First step: let us prove the following upper bound on the numerator

$$
Q(x) \leq \exp \left(C_{\alpha, \bar{N}} x^{\alpha}\right) .
$$

We split $Q(x)$ into two parts

$$
Q_{1}(x):=\prod_{\substack{\sigma \in \Lambda \\ \sigma<\lambda}}\left(1+\frac{x}{\sigma}\right) \quad \text { and } \quad Q_{2}(x):=\prod_{\substack{\sigma \in \Lambda \\ \sigma>\lambda}}\left(1+\frac{x}{\sigma}\right),
$$


and by using (3.5) we have

$$
Q_{1}(x)=\prod_{\substack{\sigma \in \Lambda \\ \sigma<x}}\left(1+\frac{x}{\sigma}\right) \quad \text { and } \quad Q_{2}(x)=\prod_{\substack{\sigma \in \Lambda \\ \sigma>x}}\left(1+\frac{x}{\sigma}\right),
$$

For the first term, we have $1 \leq x / \sigma$. Thus, from Lemma 3.1 and 1.20 , it comes that

$$
\log Q_{1}(x) \leq \sum_{\substack{\sigma \in \Lambda \\ \sigma<x}} \log \left(\frac{2 x}{\sigma}\right)=N(x) \log 2+\int_{0}^{x} \frac{1}{t} N(t) d t \leq \bar{N}(\log 2) x^{\alpha}+\frac{\bar{N}}{\alpha} x^{\alpha} .
$$

For the second term, from Lemma 3.2 , we have

$$
Q_{2}(x) \leq \prod_{\substack{\sigma \in \Lambda \\ \sigma>x}} e^{\frac{x}{\sigma}}=\exp \left(x \sum_{\substack{\sigma \in \Lambda \\ \sigma>x}} \frac{1}{\sigma}\right)=e^{x R(x)} \leq \exp \left(\frac{\bar{N}}{1-\alpha} x^{\alpha}\right) .
$$

Second step: we now prove the following lower bound on $D(x)$

$$
D(x) \geq \exp \left(-C_{\alpha, \bar{N}}(1+\log (x / \gamma)) x^{\alpha}\right)\left(\frac{\rho_{\lambda}}{2 x+\gamma}\right)^{c_{\lambda}^{\gamma}},
$$

if $N$ satisfies 1.20 and

$$
D(x) \geq \exp \left(-C_{\alpha, \bar{N}}(1-\log \gamma) x^{\alpha}\right)\left(\frac{\rho_{\lambda}}{2 x+\gamma}\right)^{c_{\lambda}^{\gamma}},
$$

if $N$ satisfies (3.2).

To this end, we study the five following terms

$$
\begin{gathered}
D_{1}(x):=\prod_{\substack{\sigma \in \Lambda \\
\sigma \leq x / 2}}\left|1-\frac{x}{\sigma}\right|, \quad D_{2}(x):=\prod_{\substack{\sigma \in \Lambda \\
x / 2<\sigma \leq x-\gamma / 2}}\left|1-\frac{x}{\sigma}\right|, \quad D_{3}(x):=\prod_{\substack{\sigma \in \Lambda, \sigma \neq \lambda \\
x-\gamma / 2<\sigma \leq x+\gamma / 2}}\left|1-\frac{x}{\sigma}\right|, \\
D_{4}(x):=\prod_{\substack{\sigma \in \Lambda \\
x+\gamma / 2<\sigma \leq 2 x}}\left|1-\frac{x}{\sigma}\right| \text { and } D_{5}(x):=\prod_{\substack{\sigma \in \Lambda \\
\sigma>2 x}}\left|1-\frac{x}{\sigma}\right| .
\end{gathered}
$$

The only difference between assumptions 1.20$)$ and $(3.2)$ will appear in the estimates of $D_{2}$ and $D_{4}$.

- For any $\sigma \leq \frac{x}{2}$, we have $\left|1-\frac{x}{\sigma}\right| \geq 1$ and thus $D_{1}(x) \geq 1$.

- To treat $D_{5}(x)$, we notice that

$$
1-u \geq e^{-2 u}, \quad \forall u \in\left[0, \frac{1}{2}\right] .
$$

Thus,

$$
D_{5}(x) \geq \exp \left(-2 x \sum_{\substack{\sigma \in \Lambda \\ \sigma>2 x}} \frac{1}{\sigma}\right) \geq e^{-2 x R(2 x)} .
$$

Then, Lemma 3.2 implies

$$
D_{5}(x) \geq \exp \left(-\frac{\bar{N}}{1-\alpha}(2 x)^{\alpha}\right) .
$$


- We now turn to the term $D_{3}(x)$. Thanks to (3.6) we have

$$
D_{3}(x):=\prod_{\substack{\sigma \in \Lambda, \sigma \neq \lambda \\ x-\gamma / 2<\sigma<x+\gamma / 2}}\left|1-\frac{x}{\sigma}\right| .
$$

Recall that $\rho_{\lambda}$ and $c_{\lambda}^{\gamma}$ are defined by (1.17) and (1.18). In particular, we have $|\lambda-\sigma| \geq \rho_{\lambda}$ and by assumption $|\lambda-x| \leq \rho_{\lambda} / 2$, so that we finally have

$$
|x-\sigma| \geq \rho_{\lambda} / 2, \quad \forall \sigma \in \Lambda, \sigma \neq \lambda .
$$

It follows that

$$
D_{3}(x) \geq \prod_{\substack{\sigma \in \Lambda, \sigma \neq \lambda \\ \sigma \in(x-\gamma / 2, x+\gamma / 2)}} \frac{\rho_{\lambda}}{2(x+\gamma / 2)} .
$$

There is now two subcases:

- If $\frac{\rho_{\lambda}}{2(x+\gamma / 2)} \leq 1$, then we can use that $(x-\gamma / 2, x+\gamma / 2) \subset(\lambda-\gamma, \lambda+\gamma)$ (because we assumed that $|x-\lambda| \leq \gamma / 2$ ) to obtain the bound from below

$$
D_{3}(x) \geq \prod_{\substack{\sigma \in \Lambda, \sigma \neq \lambda \\ \sigma \in(\lambda-\gamma, \lambda+\gamma)}} \frac{\rho_{\lambda}}{2(x+\gamma / 2)}=\left(\frac{\rho_{\lambda}}{2 x+\gamma}\right)^{c_{\lambda}^{\gamma}},
$$

by definition of $c_{\lambda}^{\gamma}$.

- If $\frac{\rho_{\lambda}}{2(x+\gamma / 2)}>1$, then in particular $\gamma<\rho_{\lambda}$ and we deduce that, we necessarily have $c_{\lambda}^{\gamma}=0$, so that (3.7) is still valid and actually reduces to $D_{3}(x) \geq 1$.

- The last terms $D_{2}(x)$ and $D_{4}(x)$ are dealt with in the exact same way. Let us detail the treatment of $D_{4}$. From Lemma 3.1 it comes that

$$
\begin{aligned}
\log D_{4}(x) & \geq \sum_{\substack{\sigma \in \Lambda \\
x+\gamma / 2<\sigma \leq 2 x}} \log \left(\frac{\sigma-x}{2 x}\right) \\
& =N(2 x) \log (1 / 2)-N(x+\gamma / 2) \log (\gamma / 4 x)-\int_{x+\gamma / 2}^{2 x} \frac{1}{t-x} N(t) \mathrm{d} t \\
& =-(\log 2) N(2 x)+N(x+\gamma / 2) \log (4 x / \gamma)-\int_{\gamma / 2}^{x} \frac{1}{u} N(u+x) \mathrm{d} u .
\end{aligned}
$$

Notice that,

$$
\int_{\gamma / 2}^{x} \frac{1}{u} d t=\log \left(\frac{2 x}{\gamma}\right)
$$

Thus,

$$
\begin{aligned}
\log D_{4}(x) \geq-(\log 2) N(2 x)+ & (\log 2) N(x+\gamma / 2) \\
& -\int_{\gamma / 2}^{x} \frac{1}{u}(N(u+x)-N(\gamma / 2+x)) \mathrm{d} u .
\end{aligned}
$$

The difference between assumptions $(1.20)$ and $(3.2)$ appears in the estimate of

$$
N(u+x)-N(\gamma / 2+x)
$$


- Assume first, that $N$ satisfies 1.20 . Formula (3.4) implies

$$
N(u+x)-N(\gamma / 2+x) \leq(u+x) R(\gamma / 2+x) \leq 2 x R(x),
$$

which leads to

$$
\log D_{4}(x) \geq-(\log 2) N(2 x)+(\log 2) N(x+\gamma / 2)-2 x R(x) \log (2 x / \gamma) .
$$

Finally, from 1.20 and Lemma 3.2 , we deduce that

$$
\log D_{4}(x) \geq-(\log 2) \bar{N}(2 x)^{\alpha}-2 \frac{\bar{N}}{1-\alpha} x^{\alpha} \log \left(\frac{2 x}{\gamma}\right)
$$

which ends the proof.

- Assume now that $N$ satisfies 3.2 . Then,

$$
\begin{aligned}
|N(x+u)-N(x+\gamma / 2)| & \leq 2 C+\bar{N}\left|(x+u)^{\alpha}-(x+\gamma / 2)^{\alpha}\right| \\
& \leq 2 C+\bar{N}|u-\gamma / 2|^{\alpha} \\
& \leq 2 C+\bar{N}|u|^{\alpha} .
\end{aligned}
$$

Integrating this inequality we obtain

$$
\begin{aligned}
\int_{\gamma / 2}^{x} \frac{1}{u}(N(x+u)-N(x+\gamma / 2)) \mathrm{d} u & \leq 2 C \log \left(\frac{2 x}{\gamma}\right)+\bar{N} \int_{\gamma / 2}^{x}|u|^{\alpha-1} \\
& \leq 2 C \log \left(\frac{2 x}{\gamma}\right)+\frac{\bar{N}}{\alpha} x^{\alpha} .
\end{aligned}
$$

Getting back to 3.8 , we obtain

$$
\log D_{4}(x) \geq-(\log 2) N(2 x)-2 C \log (2 x / \gamma)-\frac{\bar{N}}{\alpha} x^{\alpha} .
$$

Using again 3.2 ends the proof.

\subsubsection{Estimates of derivatives of Blaschke product.}

We now prove estimates similar to Proposition 3.1 but on derivatives of the inverse of Blaschke products.

Proposition 3.2. Let $\alpha \in(0,1)$ and $\bar{N}>0$. Let $\Lambda$ be a family such that its counting function $N$ satisfies 1.20 .

For any $\lambda \in \Lambda$, and any $k \geq 1$, we have the estimate

$$
\begin{array}{r}
\left|\left(\frac{1}{W_{\lambda}^{\Lambda}}\right)^{(k)}(\lambda)\right| \leq k !\left(\frac{\lambda+5}{\rho_{\lambda}}\right)^{c_{\lambda}^{\gamma}}\left(\frac{2}{\min \left(\gamma, \rho_{\lambda}\right)}\right)^{k} \exp \left(C_{\alpha, \bar{N}}\left(1+\log \left(\frac{\lambda}{\gamma}\right)\right) \lambda^{\alpha}\right), \\
\forall \gamma \in(0,2),
\end{array}
$$

where $C_{\alpha, \bar{N}}>0$ only depends on the parameters $\alpha$ and $\bar{N}$.

Remark 3.2. As detailed for Proposition 3.1, the same estimate as the one given in Proposition 3.2 holds without the $\log (\lambda)$ term in the exponential if one replaces assumption 1.20 by 3.2 . 
Proof. The proof relies on the Cauchy formula and Proposition 3.1. Let $\Gamma_{\lambda}$ be the circle centered at $\lambda$ and of radius $r_{\lambda}:=\frac{\min \left(\gamma, \rho_{\lambda}\right)}{2}$

$$
\Gamma_{\lambda}:=\left\{z \in \mathbb{C},|z-\lambda|=\frac{\min \left(\gamma, \rho_{\lambda}\right)}{2}\right\} .
$$

By definition of $\rho_{\lambda}$ the associated closed disk $\bar{D}_{\lambda}$ does not contain any other element of $\Lambda \backslash\{\lambda\}$ and therefore $\frac{1}{W_{\lambda}^{\Lambda}}$ is holomorphic in a neighborhood of $\bar{D}_{\lambda}$, and we can apply the Cauchy formula to get

$$
\left(\frac{1}{W_{\lambda}^{\Lambda}}\right)^{(k)}(\lambda)=\frac{k !}{2 i \pi} \int_{\Gamma_{\lambda}} \frac{\frac{1}{W_{\lambda}^{\Lambda}(z)}}{(z-\lambda)^{k+1}} \mathrm{~d} z .
$$

It follows that

$$
\left|\left(\frac{1}{W_{\lambda}^{\Lambda}}\right)^{(k)}(\lambda)\right| \leq \frac{k !}{r_{\lambda}^{k}} \sup _{z \in \Gamma_{\lambda}}\left|\frac{1}{W_{\lambda}^{\Lambda}(z)}\right|
$$

Thus, we are reduced to estimate $\frac{1}{W_{\lambda}^{\Lambda}}$ on $\Gamma_{\lambda}$.

For any $z \in \mathbb{C}^{+}:=\{z \in \mathbb{C}: \Re(z)>0\}$, since $|\lambda-\Re z| \leq|\lambda+\Re z|$, notice that

$$
\left|\frac{\lambda-z}{\lambda+z}\right|^{2}=\frac{(\lambda-\Re z)^{2}+(\Im z)^{2}}{(\lambda+\Re z)^{2}+(\Im z)^{2}} \geq \frac{(\lambda-\Re z)^{2}}{(\lambda+\Re z)^{2}} .
$$

Thus, we obtain, for any $z \in \mathbb{C}^{+}$

$$
\left|W_{\lambda}^{\Lambda}(z)\right| \geq\left|W_{\lambda}^{\Lambda}(\Re z)\right|
$$

Notice that, for any $\lambda \in \Lambda$, thanks to (1.17), one has $\lambda \geq \rho_{\lambda}$ and thus $\Gamma_{\lambda} \subset \mathbb{C}^{+}$. From 3.9 , we get

$$
\left|\left(\frac{1}{W_{\lambda}^{\Lambda}}\right)^{(k)}(\lambda)\right| \leq \frac{k !}{r_{\lambda}^{k}} \sup _{x \in\left[\lambda-r_{\lambda}, \lambda+r_{\lambda}\right]}\left|\frac{1}{W_{\lambda}^{\Lambda}(x)}\right|
$$

By definition of $r_{\lambda}$ we can apply Proposition 3.1 for every $x \in\left[\lambda-r_{\lambda}, \lambda+r_{\lambda}\right]$. Then, using that $r_{\lambda} \leq 1$ (since we assumed $\gamma<2$ ), the claim follows.

\subsection{Construction of biorthogonal families.}

\subsubsection{A biorthogonal family to the exponentials.}

In this section, we prove Theorem 1.3 . We start with the following construction of a biorthogonal family to the exponentials in infinite time.

Proposition 3.3. Let $\alpha \in(0,1), \bar{N}>0$ and let $\Lambda \subset(0,+\infty)$ be a countable family whose counting function $N$ satisfies 1.20 .

Then, there exists a family of functions $\left(q_{\lambda, \infty}\right)_{\lambda \in \Lambda} \subset L^{2}(0,+\infty)$ which is biorthogonal to the exponential functions

$$
\left\{t \mapsto e^{-\lambda t} ; \lambda \in \Lambda\right\}
$$

that is

$$
\int_{0}^{+\infty} e^{-\lambda t} q_{\mu, \infty}(t) \mathrm{d} t=\delta_{\lambda, \mu}, \quad \forall \lambda, \mu \in \Lambda,
$$


and that satisfies for any $\lambda \in \Lambda$, the estimate

$$
\left\|q_{\lambda, \infty}\right\|_{L^{2}(0,+\infty)} \leq C_{\alpha, \bar{N}}\left(\frac{\lambda+\gamma}{\rho_{\lambda}}\right)^{c_{\lambda}^{\gamma}} \exp \left(C_{\alpha, \bar{N}}(1+\log (\lambda / \gamma)) \lambda^{\alpha}\right), \quad \forall \gamma>0,
$$

where $C_{\alpha, \bar{N}}$ only depend on the parameters $\alpha$ and $\bar{N}$, and $\rho_{\lambda}, c_{\lambda}^{\gamma}$ are defined in (1.17) and 1.18 respectively.

REMARK 3.3. As detailed for Proposition 3.1. the same estimate as (3.11) holds without the $\log (\lambda)$ term in the exponential if one replaces assumption (1.20) by (3.2).

Proof. The proof rely on the isometry induced by the Laplace transform

$$
\begin{aligned}
\mathcal{L}: L^{2}(0,+\infty ; \mathbb{C}) & \rightarrow \mathbf{H}^{2}\left(\mathbb{C}^{+}\right) \\
f & \mapsto\left(F: \lambda \mapsto \int_{0}^{+\infty} f(t) e^{-\lambda t} \mathrm{~d} t\right)
\end{aligned}
$$

onto the Hardy space $\mathbf{H}^{2}\left(\mathbb{C}^{+}\right)$endowed with the norm

$$
\|F\|_{\mathbf{H}^{2}\left(\mathbb{C}^{+}\right)}^{2}=\int_{-\infty}^{+\infty}|F(i y)|^{2} \mathrm{~d} y .
$$

For more details about this isometry we refer to [28, p. 19-20] and the references therein.

For any given $\lambda \in \Lambda$, we define

$$
J_{\lambda}(z):=\frac{1+\lambda}{W_{\lambda}^{\Lambda}(\lambda)} \frac{W_{\lambda}^{\Lambda}(z)}{1+z}, \quad \forall z \in \mathbb{C}^{+},
$$

which is clearly holomorphic in $\mathbb{C}^{+}$. Moreover, since $W_{\lambda}^{\Lambda}$ satisfies

$$
\left|W_{\lambda}^{\Lambda}(i y)\right|=1, \quad \text { for any } y \in \mathbb{R},
$$

we get that $J_{\lambda} \in \mathbf{H}^{2}\left(\mathbb{C}^{+}\right)$and that

$$
\left\|J_{\lambda}\right\|_{\mathbf{H}^{2}\left(\mathbb{C}^{+}\right)}=\left(\int_{-\infty}^{+\infty} \frac{1}{1+y^{2}} \mathrm{~d} y\right)^{\frac{1}{2}} \frac{1+\lambda}{\left|W_{\lambda}^{\Lambda}(\lambda)\right|}=\frac{\sqrt{\pi}(1+\lambda)}{\left|W_{\lambda}^{\Lambda}(\lambda)\right|}
$$

By Proposition 3.1. we finally obtain the estimate

$$
\left\|J_{\lambda}\right\|_{\mathbf{H}^{2}\left(\mathbb{C}^{+}\right)} \leq C_{\alpha, \bar{N}}\left(\frac{\lambda+\gamma}{\rho_{\lambda}}\right)^{c_{\lambda}^{\gamma}} \exp \left(C_{\alpha, \bar{N}}(1-\log \lambda / \gamma) \lambda^{\alpha}\right) .
$$

We define $q_{\lambda, \infty}:=\Re\left(\mathcal{L}^{-1}\left(J_{\lambda}\right)\right)$ so that, by the isometry property of the Laplace transform, the expected $L^{2}$ bound for $q_{\lambda, \infty}$ holds. Moreover, by the properties of $W_{\lambda}^{\Lambda}$, we have

$$
J_{\lambda}(\lambda)=1, \quad \text { and } \quad J_{\lambda}(\mu)=0, \quad \forall \mu \in \Lambda \backslash\{\lambda\} .
$$

This translates, thanks to the Laplace transform definition, into the expected orthogonality conditions

$$
\int_{0}^{+\infty} q_{\lambda, \infty}(t) e^{-\mu t} \mathrm{~d} t=\delta_{\lambda, \mu}, \quad \mu \in \Lambda .
$$


The proof is complete.

For $T \in(0,+\infty]$, let us now introduce the spaces

$$
A(\Lambda, T)=\overline{\operatorname{Span}\left(t \mapsto e^{-\lambda t}, \lambda \in \Lambda\right)} L^{2}(0, T) .
$$

We will use the following general result that is a consequence of the study of Remeztype inequalities in Müntz spaces that can be found for instance in [12, 13]. A detailed proof is proposed in [14].

Theorem 3.1. Let $\alpha \in(0,1), \bar{N}>0$ and $\Lambda \subset(0,+\infty)$ be a family whose counting function satisfies 1.20 . For any $T>0$, there exists a $C_{\alpha, \bar{N}, T}>0$ depending only on $\alpha, \bar{N}$ and $T$ such that

$$
\|f\|_{L^{2}(0,+\infty)} \leq C_{\alpha, \bar{N}, T}\|f\|_{L^{2}(0, T)}, \quad \forall f \in A(\Lambda, \infty) .
$$

The invertibility of the restriction map on $A(\Lambda, \infty)$ was already proven for instance in [28] and uniform estimates (with respect to different parameters) for the above constant were proved in [21, Theorem 1.3] under an additional gap condition. Here, we need uniform bounds with respect to the counting function and we do not require any gap condition. This is why we will use the estimate given in Theorem 3.1

We now have all the ingredients to prove Theorem 1.3 .

Proof (of Theorem 1.3). Let $\left(q_{\lambda, \infty}\right)_{\lambda \in \Lambda}$ be the biorthogonal family to the exponentials in $L^{2}(0,+\infty)$ given by Proposition 3.3 . We start by projecting those functions onto the closed subspace $A(\Lambda, \infty)$ by setting

$$
\tilde{q}_{\lambda}:=\operatorname{Proj}_{A(\Lambda, \infty)} q_{\lambda, \infty}, \quad \forall \lambda \in \Lambda .
$$

By construction we have, for any $\mu \in \Lambda$,

$$
\int_{0}^{+\infty} \tilde{q}_{\lambda}(t) e^{-\mu t} \mathrm{~d} t=\int_{0}^{+\infty} q_{\lambda, \infty}(t) e^{-\mu t} \mathrm{~d} t=\delta_{\lambda, \mu},
$$

and moreover $\left\|\tilde{q}_{\lambda}\right\|_{L^{2}(0, \infty)} \leq\left\|q_{\lambda, \infty}\right\|_{L^{2}(0, \infty)}$. Therefore $\left(\tilde{q}_{\lambda}\right)_{\lambda}$ is also a biorthogonal family satisfying the same bounds as $\left(q_{\lambda, \infty}\right)_{\lambda}$ but it has the additional property to be included in the set $A(\Lambda, \infty)$.

For a finite given $T>0$, let us define the restriction operator

$$
R_{\Lambda, T}: f \in A(\Lambda, \infty) \mapsto f_{\mid(0, T)} \in A(\Lambda, T) .
$$

By Theorem 3.1, we know that it is invertible and that the following uniform bound holds

$$
\left\|R_{\Lambda, T}^{-1}\right\| \leq C_{\alpha, \bar{N}, T}
$$

therefore the adjoint $\left(R_{\Lambda, T}^{-1}\right)^{*}$ is an operator from $A(\Lambda, \infty)$ onto $A(\Lambda, T)$ that satisfies the same bound.

Then, setting

$$
q_{\lambda}:=\left(R_{\Lambda, T}^{-1}\right)^{*} \tilde{q}_{\lambda}, \quad \forall \lambda \in \Lambda,
$$

we see that the biorthogonality property in $L^{2}(0, T)$ is guaranteed as well as the expected bounds. $\square$ 


\subsubsection{A more general biorthogonal family.}

The previous construction can be extended to prove the existence and suitable estimates of a biorthogonal family in $L^{2}(0, T)$ to

$$
\left\{t \mapsto(-t)^{j} e^{-\lambda t} ; j \in \llbracket 0, d \rrbracket, \lambda \in \Lambda\right\},
$$

as detailed in the following theorem.

Theorem 3.2. Let $\alpha \in(0,1), \bar{N}>0, d \in \mathbb{N}$, and let $\Lambda \subset(0,+\infty)$ be a countable family whose counting function $N$ satisfies 1.20 .

Then, there exists a family of functions $\left(q_{\lambda, T}^{l}\right)_{l \in \llbracket 0, d \rrbracket, \lambda \in \Lambda} \subset L^{2}(0,+\infty)$ which has the following biorthogonality property

$$
\int_{0}^{T}(-t)^{j} e^{-\lambda t} q_{\mu, T}^{l}(t) \mathrm{d} t=\delta_{\lambda, \mu} \delta_{j, l}, \quad \forall \lambda, \mu \in \Lambda, \quad \forall j, l \in \llbracket 0, d \rrbracket,
$$

and that satisfies for any $\lambda \in \Lambda$ and $j \in \llbracket 0, d \rrbracket$, the estimates

$$
\begin{aligned}
\left\|q_{\lambda, T}^{j}\right\|_{L^{2}(0, T)} \leq C_{\alpha, \bar{N}, T, d} & \left(\frac{\lambda+5}{\rho_{\lambda}}\right)^{(d+1) c_{\lambda}^{\gamma}}\left(\frac{2}{\min \left(\gamma, \rho_{\lambda}\right)}\right)^{d+1} \\
& \times \exp \left(C_{\alpha, \bar{N}, d}(1+\log (\lambda / \gamma)) \lambda^{\alpha}\right), \quad \forall \gamma \in(0,2),
\end{aligned}
$$

where $C_{\alpha, \bar{N}, d}$ and $C_{\alpha, \bar{N}, T, d}$ only depend on the parameters $\alpha, \bar{N}, d$ (and $T$ ).

Remark 3.4. As detailed for Proposition 3.1. the same estimate as (3.15) holds without the $\log (\lambda)$ term in the exponential if one replaces assumption (1.20) by (3.2).

Proof. The proof follows the same line as the one of Theorem 1.3. We only detail how to adapt Proposition 3.3 .

For any $\lambda \in \Lambda$ and any $l \in \llbracket 0, d \rrbracket$, we are looking for a function $J_{\lambda}^{l} \in \mathbf{H}^{2}\left(\mathbb{C}^{+}\right)$ satisfying

1. $\forall \sigma \in \Lambda \backslash\{\lambda\}, \forall p \in \llbracket 0, d \rrbracket,\left(J_{\lambda}^{l}\right)^{(p)}(\sigma)=0$,

2. $\forall p \in \llbracket 0, d \rrbracket,\left(J_{\lambda}^{l}\right)^{(p)}(\lambda)=\delta_{p, l}$,

with suitable estimates of its norm. Notice that condition (3.14) amounts to:

$$
\left[\mathcal{L}\left(q_{\lambda}^{l}\right)\right]^{(p)}(\sigma)=\delta_{\lambda, \sigma} \delta_{p, l}
$$

which is given by conditions 1 and 2 .

Let us look for $J_{\lambda}^{l}$ in the following form

$$
J_{\lambda}^{l}(z):=P_{\lambda}^{l}(z)\left(\frac{W_{\lambda}^{\Lambda}(z)}{\beta(z)}\right)^{d+1}
$$

where $\beta(z)=1+z$ and $P_{\lambda}^{l}$ is a polynomial of degree $d$ to be determined so that condition 2 is satisfied. Notice that the term $\left(W_{\lambda}^{\Lambda}\right)^{d+1}$ ensures that condition 1 holds, whereas the term $\frac{1}{\beta(z)^{d+1}}=\frac{1}{(1+z)^{d}}$ makes $J_{\lambda}^{l}$ integrable on $i \mathbb{R}$ as we shall see.

Let us now determine the polynomial $P_{\lambda}^{l}$. For any $k \in \llbracket 0, d \rrbracket$, this polynomial 
should satisfy

$$
\begin{aligned}
\left(P_{\lambda}^{l}\right)^{(k)}(\lambda) & =\left[J_{\lambda}^{l} \times\left(\frac{\beta}{W_{\lambda}^{\Lambda}}\right)^{d+1}\right]^{(k)}(\lambda) \\
& =\sum_{p=0}^{k}\left(\begin{array}{l}
k \\
p
\end{array}\right)\left(J_{\lambda}^{l}\right)^{(p)}(\lambda)\left[\left(\frac{\beta}{W_{\lambda}^{\Lambda}}\right)^{d+1}\right]^{(k-p)}(\lambda) \\
& =\sum_{p=0}^{k}\left(\begin{array}{l}
k \\
p
\end{array}\right) \delta_{p, l}\left[\left(\frac{\beta}{W_{\lambda}^{\Lambda}}\right)^{d+1}\right]^{(k-p)}(\lambda) \\
& =\left\{\begin{array}{l}
k \\
l
\end{array}\right)\left[\left(\frac{\beta}{W_{\lambda}^{\Lambda}}\right)^{d+1}\right]^{(k-l)}(\lambda) \quad \text { if } k \geq l, \\
0 & \text { if } k<l .
\end{aligned}
$$

Thus, setting

$$
P_{\lambda}^{l}(z)=\sum_{k=l}^{d}\left(\begin{array}{l}
k \\
l
\end{array}\right)\left[\left(\frac{\beta}{W_{\lambda}^{\Lambda}}\right)^{d+1}\right]^{(k-l)}(\lambda) \frac{(z-\lambda)^{k}}{k !}
$$

ensures condition 2. We now turn to the estimate of $\left\|J_{\lambda}^{l}\right\|_{\mathbf{H}^{2}\left(\mathbb{C}^{+}\right)}$. First, recall that for all $y \in \mathbb{R}$, we have $\left|W_{\lambda}^{\Lambda}(i y)\right|=1$. Therefore,

$$
\left\|J_{\lambda}^{l}\right\|_{\mathbf{H}^{2}\left(\mathbb{C}^{+}\right)}^{2}=\int_{-\infty}^{\infty}\left|J_{\lambda}^{l}(i y)\right|^{2} \mathrm{~d} y=\int_{-\infty}^{\infty}\left|\frac{P_{\lambda}^{l}(i y)}{(1+i y)^{d+1}}\right|^{2} \mathrm{~d} y
$$

Note that, according to 3.16 , there exists $C_{d}>0$ such that, for any $z \in \mathbb{C}$,

$$
\left|\left(P_{\lambda}^{l}\right)(z)\right| \leq C_{d}(1+|z|)^{d}(1+\lambda)^{2 d+1} \sum_{k=0}^{d-l}\left|\left[\frac{1}{\left(W_{\lambda}^{\Lambda}\right)^{d+1}}\right]^{(k)}(\lambda)\right| .
$$

Hence,

$$
\left\|J_{\lambda}^{l}\right\|_{\mathbf{H}^{2}\left(\mathbb{C}^{+}\right)} \leq C_{d}(1+\lambda)^{2 d+1} \sum_{k=0}^{d-l}\left|\left[\frac{1}{\left(W_{\lambda}^{\Lambda}\right)^{d+1}}\right]^{(k)}(\lambda)\right| .
$$

Then, from Leibniz rule and Proposition 3.2 , we obtain (3.15).

As in the proof of Theorem 1.3 , the desired biorthogonal family in $L^{2}(0, T ; \mathbb{R})$ will follow from the suitable version of Theorem 3.1 adapted to the new family of functions we consider here, see the details in [14].

\section{Application to cascade systems of Grushin equations.}

We gather the spectral analysis done in Section 2 and the general construction of biorthogonal family proved in Section 3 to prove Theorem 1.1. Then, in Section 4.2 . we extend these results to the cascade system of coupled Grushin equations 1.22 . 


\subsection{Minimal null control time of Grushin equation.}

In this section, we prove Theorem 1.1. From the strategy detailed in Section 1. we want to prove that for $T>\frac{a^{2}}{2}$ the system 1.4 is null controllable with a control $u_{n}$ uniformly bounded with respect to $n$. The same arguments as the one of [8, Proposition 4.1] (see also [8, Proposition 3.1]) based on cut-off arguments prove that it is sufficient to consider the case $\omega=(a, 1) \times(0,1)$.

Recall that from (1.6), the control

$$
u_{n}(t, x)=-\sum_{k \in \mathbb{N}^{*}} e^{-\lambda_{k, n} T}\left\langle f_{n}^{0}, g_{k, n}\right\rangle_{L^{2}(-1,1)} \frac{g_{k, n}(x)}{\left\|g_{k, n}\right\|_{L^{2}(a, 1)}^{2}} q_{k, n}(T-t),
$$

formally solves the moments problem (1.5).

To deal with the biorthogonal family $\left(q_{k, n}\right)_{k \in \mathbb{N}^{*}}$, we prove the following uniform bound on the counting function.

Proposition 4.1. There exists $C>0$, such that,

$$
\lambda_{k, n} \geq C k^{2}, \quad \forall k, n \in \mathbb{N}^{*} .
$$

Thus, there exists $C>0$ such that, the counting function $N_{n}$ of the family $\Lambda_{n}$ (see (1.16) satisfies

$$
N_{n}(r) \leq C \sqrt{r}, \quad \forall r>0, \forall n \in \mathbb{N}^{*}
$$

Proof. In [17, p.104, thm 11], the authors use Rayleigh's Theorem to deduce that

$$
\lambda_{k, n}=\frac{k^{2} \pi^{2}}{4}+r_{k, n}
$$

where $\left|r_{k, n}\right| \leq n^{2} \pi^{2}$ for any $n, k \in \mathbb{N}^{*}$. To prove 4.1), we consider two different situations

- if $k \geq 3 n$ then, according to 4.2 , there exists $c_{1}>0$ such that

$$
\lambda_{k, n} \geq \frac{k^{2} \pi^{2}}{4}-n^{2} \pi^{2} \geq c_{1} k^{2},
$$

- if $k<3 n$ then, according to Proposition 2.1, there exists $c_{2}>0$, such that

$$
\lambda_{k, n} \geq(2 k-1) n \pi \geq k n \geq c_{2} k^{2} .
$$

Then we set $C:=\min \left(c_{1}, c_{2}\right)$. The uniform asymptotic on $N_{n}$ follows directly.

We now get the following bound on the biorthogonal family using Theorem 1.3 . The estimate given by Theorem 1.3 will be sufficient for our purpose: in the range of frequencies for which we do not have a uniform gap, we have a poor gap-type inequality (see Proposition 1.2 but which concerns not so much frequencies (see (1.15)).

Proposition 4.2. Let $T>0$ and $\varepsilon>0$. There exists $C_{T, \varepsilon}>0$ such that, for any $n \in \mathbb{N}^{*}$, there exists a biorthogonal family $\left(q_{k, n}\right)_{k \in \mathbb{N}^{*}}$ to the exponentials associated with $\Lambda_{n}$ in $L^{2}(0, T ; \mathbb{R})$ i.e.

$$
\int_{0}^{T} e^{-\lambda_{k, n} t} q_{j, n}(t) \mathrm{d} t=\delta_{k, j}, \quad \forall k, j \in \mathbb{N}^{*},
$$


which satisfies

$$
\left\|q_{k, n}\right\|_{L^{2}(0, T)} \leq C_{T, \varepsilon} e^{\varepsilon \lambda_{k, n}}, \quad \forall k \in \mathbb{N}^{*}
$$

Proof. Let $\varepsilon>0$. Let $\tau \in\left[\frac{3}{4}, 1\right)$ be such that

$$
3(1-\tau) \leq \frac{\varepsilon}{2},
$$

and $\gamma_{\tau}$ the constant given by Corollary 1.2 .

Using Theorem 1.3 with the parameter $\gamma_{\tau}$ and Proposition 4.1. there exists such a biorthogonal family which satisfies

$$
\left\|q_{k, n}\right\|_{L^{2}(0, T)} \leq C_{T} \exp \left(C\left(1-\log \gamma_{\tau}+\log \lambda_{k, n}\right) \sqrt{\lambda_{k, n}}\right)\left(\frac{\lambda_{k, n}+\gamma_{\tau}}{\rho_{k, n}}\right)^{c_{k, n}^{\gamma_{\tau}}} .
$$

We now consider two cases.

- If $\lambda_{k, n} \in L_{\tau, n} \cup U_{n}$, then, from Corollary 1.2, it comes that $\rho_{k, n} \geq \gamma_{\tau}$ and thus $c_{k, n}^{\gamma_{\tau}}=0$. Then, we obtain

$$
\left\|q_{k, n}\right\|_{L^{2}(0, T)} \leq C_{T} \exp \left(C\left(1-\log \gamma_{\tau}+\log \lambda_{k, n}\right) \sqrt{\lambda_{k, n}}\right),
$$

which ends the proof in this case.

- Otherwise, $\lambda_{k, n} \in M_{\tau, n}$. From Proposition 1.2 and Proposition 2.1, we obtain

$$
\rho_{k, n} \geq \frac{\sqrt{\lambda_{k, n}}}{2} \exp \left(-\frac{8}{3} \sqrt{\lambda_{k, n}}\right) \geq \exp \left(-3 \sqrt{\lambda_{k, n}}\right) .
$$

From Corollary 1.2, we have $\operatorname{dist}\left(L_{\tau, n}, U_{n}\right) \geq \gamma_{\tau}$. Together with 1.15 and 1.13, this implies

$$
c_{k, n}^{\gamma_{\tau}} \leq \# M_{\tau, n}+1 \leq(1-\tau) \frac{n \pi}{2}+2 \leq(1-\tau) \sqrt{\lambda_{k, n}}+2 .
$$

From 4.3], it comes that

$$
\left(\frac{1}{\rho_{k, n}}\right)^{c_{k, n}^{\gamma_{\tau}}} \leq \exp \left(3(1-\tau) \lambda_{k, n}+6 \sqrt{\lambda_{k, n}}\right) \leq \exp \left(\frac{\varepsilon}{2} \lambda_{k, n}+6 \sqrt{\lambda_{k, n}}\right) .
$$

Finally, this implies

$$
\left\|q_{k, n}\right\|_{L^{2}(0, T)} \leq C_{T} \exp \left(C\left(1-\log \gamma_{\tau}+\log \left(\lambda_{k, n}+\gamma_{\tau}\right)\right) \sqrt{\lambda_{k, n}}\right) \exp \left(\frac{\varepsilon}{2} \lambda_{k, n}\right),
$$

which ends the proof. $\square$

REMARK 4.1. As mentioned in the beginning of Section 1.4 .3 , estimate $(4.4)$ is the key point to obtain the bound on the biorthogonal family given in Proposition 4.2. The very weak gap information coming from Proposition 1.2 is somehow compensated by the fact that this information does not concern too many eigenvalues (see (1.15)).

We can now prove Theorem 1.1 .

Proof (of Theorem 1.1). Let $T>\frac{a^{2}}{2}$. Let $\varepsilon>0$ and $\eta>0$ be such that

$$
T>\frac{a^{2}}{2}+\frac{\eta}{2}+\varepsilon
$$


From Proposition 1.1 it comes that

$$
\left\|g_{k, n}\right\|_{L^{2}(a, 1)}^{2} \geq C_{\eta} \frac{e^{-n \pi\left(a^{2}+\eta\right)}}{n^{2} \pi^{2}} .
$$

From Proposition 2.1 it comes that

$$
\lambda_{k, n} \geq n \pi(2 k-1) \geq n \pi+2(k-1), \quad \forall k, n \in \mathbb{N}^{*} .
$$

Together with Proposition 4.2 we obtain that the control $u_{n}$ given by 1.6 satisfies

$$
\begin{aligned}
\left\|u_{n}\right\|_{L^{2}((0, T) \times(a, 1))} & \leq C_{\varepsilon, \eta}\left(\sum_{k \in \mathbb{N}^{*}} e^{-2(k-1)(T-\varepsilon)}\right) n^{2} \pi^{2} e^{-n \pi\left(T-\frac{a^{2}}{2}-\frac{\eta}{2}-\varepsilon\right)}\left\|f_{n}^{0}\right\|_{L^{2}(-1,1)} \\
& \leq C_{\varepsilon, \eta, T}\left\|f_{n}^{0}\right\|_{L^{2}(-1,1)}
\end{aligned}
$$

which ends the proof of Theorem 1.1.

4.2. Minimal null control time of cascade systems of Grushin equations. This section is dedicated to the proof of Theorem 1.4 that is the proof of null controllability in time $T>\frac{a^{2}}{2}$ of the cascade system 1.22). As in the scalar case, one of the crucial steps is the following estimate of biorthogonal families.

Proposition 4.3. Let $T>0, \varepsilon>0$ and $d \geq 2$. There exists $C_{T, \varepsilon, d}>0$ such that, for any $n \in \mathbb{N}^{*}$, there exists a biorthogonal family $\left(q_{k, n}^{l}\right)_{k \in \mathbb{N}^{*}, l \in \llbracket 0, d-1 \rrbracket}$ to

$$
\left\{t \mapsto(-t)^{l} e^{-\lambda_{k, n} t} ; k \geq 1, l \in \llbracket 0, d-1 \rrbracket\right\}
$$

in $L^{2}(0, T ; \mathbb{R})$ i.e.

$$
\int_{0}^{T}(-t)^{p} e^{-\lambda_{k, n} t} q_{j, n}^{l}(t) \mathrm{d} t=\delta_{k, j} \delta_{p, l}, \quad \forall k, j \in \mathbb{N}^{*}, \forall p, l \in \llbracket 0, d-1 \rrbracket,
$$

which satisfies

$$
\left\|q_{k, n}^{l}\right\|_{L^{2}(0, T)} \leq C_{T, \varepsilon, d} e^{\varepsilon \lambda_{k, n}}, \quad \forall k \in \mathbb{N}^{*}, \forall l \in \llbracket 0, d-1 \rrbracket .
$$

Proof. Let $\varepsilon>0$ and $d \geq 2$. Let $\tau \in\left[\frac{3}{4}, 1\right)$ be such that

$$
3 d(1-\tau) \leq \frac{\varepsilon}{2},
$$

and $\gamma_{\tau}$ the constant given by Corollary 1.2 .

The rest of the proof follows exactly the proof of Proposition 4.2 but we apply the abstract construction given in Theorem 3.2 instead of Theorem 1.3 . Again, if $\lambda_{k, n} \in L_{\tau, n} \cup U_{n}$, the estimate follows directly whereas, if $\lambda_{k, n} \in M_{\tau, n}$ the main contribution comes from the term

$$
\left(\frac{1}{\rho_{\lambda}}\right)^{d c_{\lambda}^{\gamma_{\tau}}} \leq \exp \left(3 d(1-\tau) \lambda_{k, n}+6 d \sqrt{\lambda_{k, n}}\right) \leq e^{\frac{\varepsilon}{2} \lambda_{k, n}+6 d \sqrt{\lambda_{k, n}}}
$$

where we used 4.5.

We now turn to the proof of Theorem 1.4 . 
Proof (of Theorem 1.4). Let $T>\frac{a^{2}}{2}$. Following the method presented in Section 1.3 for every $n \in \mathbb{N}^{*}$, it suffices to find a null control $u_{n}$, uniformly bounded with respect to $n$, to the following $1 \mathrm{D}$ system

$$
\begin{cases}\partial_{t} F_{n}(t, x)+\mathcal{O}_{n} F_{n}(t, x)=B_{d} u_{n}(t, x) 1_{(a, b)}(x), & (t, x) \in(0, T) \times(-1,1), \\ F_{n}(t, \pm 1)=0, & t \in(0, T), \\ F_{n}(0, x)=F_{n}^{0}(x) \in\left(L^{2}(-1,1)\right)^{d}, & x \in(-1,1),\end{cases}
$$

where we have introduced the operator $\mathcal{O}_{n}:=\mathcal{A}_{n} I_{d}+K_{d}$.

The reduction of the null controllability of cascase systems like (4.6) to a moments problem as well as its formal resolution cand be found for instance in [3, Section 5.1]. For an adaptation with a distributed control as considered in (4.6), we refer for instance to [2, Proof of Theorem 5.2].

Following these references, we consider the Hilbert basis of $L^{2}(-1,1 ; \mathbb{R})^{d}$ of generalized eigenvectors

$$
\Phi_{k, n}^{r}(x):=g_{k, n}(x) e_{r}, \quad \forall r \in \llbracket 1, d \rrbracket, k, n \in \mathbb{N}^{*},
$$

where $e_{r}$ is the $r^{\text {th }}$ vector of the canonical basis of $\mathbb{R}^{d}$ i.e.

$$
\left(\mathcal{O}_{n}^{*}-\lambda_{k, n}\right) \Phi_{k, n}^{r}=\Phi_{k, n}^{r-1},
$$

where $\Phi_{k, n}^{0}=0$. With this notation, a formal null control of 4.6 is given by

$$
u_{n}(t, x)=-\sum_{k \in \mathbb{N}^{*}} \sum_{r=1}^{d}(r-1) !\left\langle F_{n}^{0}, e^{-\mathcal{O}_{n}^{*} T} \Phi_{k, n}^{r}\right\rangle_{L^{2}(-1,1)} \frac{g_{k, n}(x)}{\left\|g_{k, n}\right\|_{L^{2}(a, b)}^{2}} q_{k, n}^{r-1}(T-t) .
$$

From 4.7), we obtain

$$
e^{-\mathcal{O}_{n}^{*} t} \Phi_{k, n}^{r}=e^{-\lambda_{k, n} t} \sum_{s=0}^{r-1} \frac{(-t)^{s}}{s !} \Phi_{k, n}^{r-s}
$$

which implies

$$
\left|\left\langle F_{n}^{0}, e^{-\mathcal{O}_{n}^{*} T} \Phi_{k, n}^{r}\right\rangle_{L^{2}(-1,1)}\right| \leq C_{d} e^{-\lambda_{k, n} T}\left\|F_{n}^{0}\right\|_{L^{2}(-1,1)} .
$$

Then, null controllability in time $T$ follows exactly as in the proof of Theorem 1.1 .

\subsection{Extension to boundary controls.}

In this section we detail how to adapt our analysis to deal with boundary controls. This leads to Theorem 1.5. First, let us consider the scalar case i.e. $d=1$.

Using a classical argument based on cut-off functions, the lack of null controllability in time $T<\frac{1}{2}$ follows directly from Theorem 1.1 .

Now, let $T>\frac{1}{2}$ and $\varepsilon>0$ be such that $T>\frac{1}{2}+2 \varepsilon$. As previously, null controllability of 1.2 is equivalent to the uniform (with respect to the parameter $n$ ) controllability of the following family of problems

$$
\begin{cases}\partial_{t} f_{n}(t, x)+\mathcal{A}_{n} f_{n}(t, x)=0, & (t, x) \in(0, T) \times(-1,1), \\ f_{n}(t,-1)=0, & t \in(0, T), \\ f_{n}(t, 1)=u_{n}(t), & t \in(0, T), \\ f_{n}(0, x)=f_{n}^{0}(x), & x \in(-1,1) .\end{cases}
$$


The moments problem equivalent to the null controllability of 4.8 now reads

$$
g_{k, n}^{\prime}(1) \int_{0}^{T} e^{-\lambda_{k, n}(T-t)} u_{n}(t) \mathrm{d} t=-\left\langle f_{n}^{0}, e^{-\lambda_{k, n} T} g_{k, n}\right\rangle_{L^{2}(-1,1)}, \quad \forall k \in \mathbb{N}^{*} .
$$

A formal solution of this countable family of equations is given by

$$
u_{n}(t)=-\sum_{k \in \mathbb{N}^{*}} e^{-\lambda_{k, n} T} \frac{\left\langle f_{n}^{0}, g_{k, n}\right\rangle_{L^{2}(-1,1)}}{g_{k, n}^{\prime}(1)} q_{k, n}(T-t),
$$

where, for any $n \in \mathbb{N}^{*},\left(q_{k, n}\right)_{k \in \mathbb{N}^{*}}$ is the biorthogonal family in $L^{2}(0, T ; \mathbb{R})$ to

$$
\left\{t \mapsto e^{-\lambda_{k, n} t} ; k \in \mathbb{N}^{*}\right\}
$$

given in Proposition 4.2. Thus, there exists $C_{T, \varepsilon}>0$ such that

$$
\left\|q_{k, n}\right\|_{L^{2}(0, T)} \leq C_{T, \varepsilon} e^{\varepsilon \lambda_{k, n}}, \quad \forall k \in \mathbb{N}^{*}, \forall n \in \mathbb{N}^{*} .
$$

The only thing left to prove unifom null controllability of 4.8 in time $T$ is to give a suitable lower bound on $\left|g_{k, n}^{\prime}(1)\right|$. This lower bound is given in the following proposition.

Proposition 4.4. For any $\varepsilon>0$, there exists $C_{\varepsilon}>0$ such that

$$
\left|g_{k, n}^{\prime}(1)\right| \geq C_{\varepsilon} e^{-\lambda_{k, n}\left(\frac{1}{2}+\varepsilon\right)}, \quad \forall k \in \mathbb{N}^{*}, \forall n \in \mathbb{N}^{*} .
$$

Proof. Using a classical argument of extension of the spatial domain, the analysis of the minimal null control time done in $[8]$ implies the null controllability in any time $T>\frac{1}{2}$ of the boundary control problem

$$
\begin{cases}\partial_{t} f(t, x, y)+\mathcal{D} f(t, x, y)=0, & (t, x, y) \in(0, T) \times \Omega \\ f(t, x, y)=0, & (t, x, y) \in(0, T) \times(\partial \Omega \backslash \Gamma) \\ f(t, x, y)=u(t, y), & (t, x, y) \in(0, T) \times \Gamma \\ f(0, x, y)=f^{0}(x, y) \in L^{2}(\Omega), & (x, y) \in \Omega,\end{cases}
$$

where $\Gamma=\Gamma_{-1} \cup \Gamma_{1}$ with $\Gamma_{ \pm 1}=\{ \pm 1\} \times[0,1]$. Thus, this problem satisfies the quantitative Fattorini-Hautus (proved in [19, Proposition 2.3] and studied in more details in [5]) which is a necessary condition for null controllability in time $T$. In particular, for any $\varepsilon>0$, there exists $C_{\varepsilon}>0$ such that every normalized eigenvector $\varphi_{\lambda}$ of $\mathcal{D}$ associated with the eigenvalue $\lambda$ satisfies

$$
\left\|\partial_{\nu} \varphi_{\lambda}\right\|_{L^{2}(\Gamma)}^{2} \geq C_{\varepsilon} \lambda e^{-2 \lambda\left(\frac{1}{2}+\varepsilon\right)} .
$$

Applying this inequality to $(x, y) \mapsto g_{k, n}(x) \phi_{n}(y)$ we obtain

$$
\left|g_{k, n}^{\prime}(-1)\right|^{2}+\left|g_{k, n}^{\prime}(1)\right|^{2} \geq C_{\varepsilon} \lambda_{k, n} e^{-2 \lambda_{k, n}\left(\frac{1}{2}+\varepsilon\right)}, \quad \forall k \in \mathbb{N}^{*}, \forall n \in \mathbb{N}^{*} .
$$

As the eigenfunctions $g_{k, n}$ are either odd or even (see Lemma 2.1) this ends the proof of Proposition 4.4 . 
Recall that, from Proposition 2.1.

$$
\lambda_{k, n} \geq n \pi(2 k-1) \geq 2 k-1, \quad \forall k \in \mathbb{N}^{*}, \forall n \in \mathbb{N}^{*} .
$$

Then, using Proposition 4.4 and estimate 4.11 in 4.10 leads to the existence of $C_{T, \varepsilon}>0$ such that

$$
\begin{aligned}
\left\|u_{n}\right\|_{L^{2}(0, T)} & \leq C_{T, \varepsilon} \sum_{k \in \mathbb{N}^{*}} e^{-\lambda_{k, n} T} e^{\lambda_{k, n}\left(\frac{1}{2}+\varepsilon\right)} e^{\varepsilon \lambda_{k, n}}\left|\left\langle f_{n}^{0}, g_{k, n}\right\rangle_{L^{2}(-1,1)}\right| \\
& \leq C_{T, \varepsilon} \sum_{k \in \mathbb{N}^{*}}\left(e^{-(2 k-1)\left(T-\frac{1}{2}-2 \varepsilon\right)}\right)\left\|f_{n}^{0}\right\|_{L^{2}(-1,1)}
\end{aligned}
$$

which ends the proof of Theorem 1.5 in the case $d=1$.

The adaptation to the minimal null control time of the cascade system 1.23 follows exactly the lines of Section 4.2 .

\section{Appendix.}

\subsection{Proof of Lemma 2.3,}

Recall that the functions $\left(G_{k}\right)_{k \geq 1}$ are normalized in $L^{2}(\mathbb{R})$ and satisfy

$$
G_{k}(x)=\frac{e^{-x^{2} / 2} H_{k}(x)}{\pi^{1 / 4} 2^{\frac{k-1}{2}} \sqrt{(k-1) !}},
$$

where $H_{k}$ is the $(k-1)^{\text {th }}$ Hermite polynomial defined by

$$
H_{k}(x)=(-1)^{k-1} e^{x^{2}} \frac{\mathrm{d}^{k-1}}{\mathrm{~d} x^{k-1}} e^{-x^{2}}, \forall k \geq 1 .
$$

These polynomials are orthogonal for the following scalar product

$$
(f, g)=\int_{\mathbb{R}} f(x) g(x) e^{-x^{2}} \mathrm{~d} x .
$$

Those functions are drawn in Figure 5.1

Let us start by showing

$$
0<G_{k}(x) \leq G_{k+1}(x), \quad \forall x \geq \sqrt{n \pi}, \forall k \in \llbracket 1, \frac{\pi}{2} n \rrbracket .
$$

One can observe this inequality in Figure 5.1

From the definition of Hermite polynomials, one can derive easily the classical recurrence relation (see for instance [29, Section 5.5])

$$
H_{k}(x)=2 x H_{k-1}(x)-2(k-2) H_{k-2}(x), \quad \forall k \geq 2 .
$$

Then, we use (5.1) to get:

$$
\begin{aligned}
G_{k}(x) & =2 x \frac{e^{-x^{2} / 2} H_{k-1}(x)}{\pi^{1 / 4} 2^{\frac{k-1}{2}} \sqrt{(k-1) !}}-\frac{2(k-2)}{\pi^{1 / 4} 2^{\frac{k-1}{2}} \sqrt{(k-1) !}} e^{-x^{2} / 2} H_{k-2}(x) \\
& =\sqrt{\frac{2}{k-1}} x G_{k-1}(x)-\frac{\sqrt{k-2}}{\sqrt{k-1}} G_{k-2}(x) .
\end{aligned}
$$




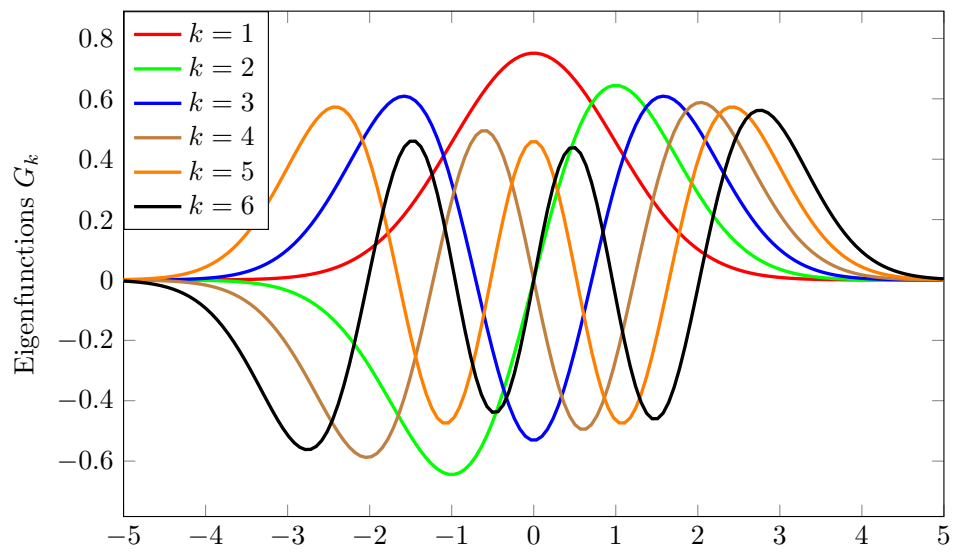

Figure 5.1: Graph of some eigenfunctions of the harmonic oscillator.

Let us fix $n \in \mathbb{N}^{*}$ and prove $(5.2)$ by induction on $k$.

Since $G_{1}(x)=\frac{e^{-x^{2} / 2}}{\pi^{1 / 4}}$ and $G_{2}(x)=\frac{x e^{-x^{2} / 2}}{\pi^{1 / 4} \sqrt{2}}$, inequality 5.2 holds for $k=1$.

Take $k \geq 2$ and suppose that 5.2 holds for $k-1$ : for any $x \geq \sqrt{n \pi}, G_{k}(x) \geq$ $G_{k-1}(x)>0$. According to the above recurrence relation, we have

$$
G_{k+1}(x)=\sqrt{\frac{2}{k}} x G_{k}(x)-\sqrt{\frac{k-1}{k}} G_{k-1}(x) .
$$

Thus,

$$
G_{k+1}(x) \geq\left(\sqrt{\frac{2}{k}} x-\sqrt{\frac{k-1}{k}}\right) G_{k}(x) .
$$

Since $x \geq \sqrt{n \pi}$, we have

$$
\sqrt{\frac{2}{k}} x-\sqrt{\frac{k-1}{k}} \geq \sqrt{\frac{2}{k}} \sqrt{n \pi}-1 \geq \sqrt{\frac{2}{k}} \sqrt{2 k}-1 \geq 1,
$$

since $k \leq \frac{\pi n}{2}$. As $G_{k}(x)>0$, this gives 5.2 .

According to inequality $(5.2)$, Lemma 2.3 is proved, if for $k_{0}:=\left\lfloor\tau n \frac{\pi}{2}\right\rfloor$, the following inequality holds

$$
G_{k_{0}}(\sqrt{n \pi}) \leq C_{1} e^{-C_{2} n} .
$$

Let us show this inequality. First, for any $k \in \mathbb{N}^{*}, G_{k}$ is decreasing in $\left[\sqrt{\mu_{k}},+\infty\right)$. Indeed, when $x \geq \sqrt{\mu_{k}}$, we have proved that $G_{k}(x)>0$. Moreover, for a such $x$, $G_{k}^{\prime \prime}(x)=\left(x^{2}-\mu_{k}\right) G_{k}(x) \geq 0$. Thus, the function $G_{k}$ is convex in $\left[\sqrt{\mu_{k}},+\infty[\right.$ and tends to zero at infinity. It follows that $G_{k}$ is decreasing in this interval.

It suffices now to show that $G_{k_{0}}\left(x_{0}\right) \leq C_{1} e^{-C_{2} n}$ for a $x_{0}$ such that $\sqrt{n \pi} \geq x_{0} \geq$ $\sqrt{\mu_{k_{0}}}$. Recall that $\mu_{k}=2 k-1$.

As $\tau \in(0,1)$, let $\alpha>0$ be such that $\cosh (\alpha)=\sqrt{\frac{1}{\tau}}$. Let $x_{0}:=\sqrt{\mu_{k_{0}}} \cosh (\alpha)$. Observe that, for any $n \in \mathbb{N}^{*}$,

$$
\sqrt{n \pi} \geq x_{0} \geq \sqrt{\mu_{k_{0}}}
$$


Then, the estimate given in [29, formula (8.22.13), p. 201] reads

$$
G_{k_{0}}\left(x_{0}\right)=\frac{\exp \left[\left(\frac{k_{0}}{2}-\frac{1}{4}\right)(2 \alpha-\sinh (2 \alpha)]\right.}{\sqrt{\pi} 2^{3 / 4}\left(k_{0}-1\right)^{1 / 4} \sqrt{\sinh (\alpha)}}\left(1+O_{\tau}\left(\frac{1}{n}\right)\right),
$$

where $O_{\tau}\left(\frac{1}{n}\right)$ only depends on $\tau$ and $n$. Recall that $\alpha>0$, so using that $2 \alpha-$ $\sinh (2 \alpha)<0$, we find, for $n \geq n_{0}$ with $n_{0}$ large enough,

$$
G_{k_{0}}\left(x_{0}\right) \leq C_{1} \exp \left(-C_{2} n\right),
$$

where $C_{1}$ et $C_{2}$ only depend on $\tau$.

REMARK 5.1. Lemma 2.3 is the only step in the whole spectral analysis that requires the introduction of the parameter $\tau$. This is what introduces a discrepancy between the analysis of low and high frequencies and led us to the generalization of biorthogonal families stated in Theorem 1.3. In the case $\tau=1$, instead of Lemma2.3. we only obtain a polynomial decay (with respect to $n$ ) of $G_{k}(x)$ in the considered range for $k$ and $x$. This decay would not be sufficient to repeat the proof of the uniform gap property fo low frequencies (see for instance (2.19).

\subsection{Proof of Lemma 2.2,}

We shall in fact prove the following slightly more general property: for any $\delta \in$ $(0,1)$, there exists an integer $n_{0}(\delta) \in \mathbb{N}^{*}$ such that

$$
\int_{\Omega_{n}} G_{k}^{2}(x) \mathrm{d} x \geq 1-\delta, \quad \forall k \leq \frac{\pi}{2} n, \quad \forall n \geq n_{0} .
$$

Recall that $\mu_{k}=2 k-1$. Let $\delta \in(0,1)$ and $\varepsilon \in\left(0, \frac{\pi}{2}\right)$ to be defined later, but depending only on $\delta$. Let $k \leq \frac{\pi}{2} n$, we have $\sqrt{n \pi} \geq \sqrt{\mu_{k}} \geq \sqrt{\mu_{k}} \cos (\varepsilon)$. Since $G_{k}^{2}$ is even,

$$
\int_{\Omega_{n}} G_{k}^{2}(x) \mathrm{d} x=2 \int_{0}^{\sqrt{n \pi}} G_{k}^{2}(x) \mathrm{d} x \geq 2 \int_{0}^{\sqrt{\mu_{k}} \cos (\varepsilon)} G_{k}^{2}(x) \mathrm{d} x .
$$

Let us make the change of variable $x=\sqrt{\mu_{k}} \cos (\phi)$ with $\phi \in\left[\varepsilon, \frac{\pi}{2}\right]$,

$$
\int_{\Omega_{n}} G_{k}^{2}(x) \mathrm{d} x \geq 2 \int_{\varepsilon}^{\frac{\pi}{2}} G_{k}^{2}\left(\sqrt{\mu_{k}} \cos (\phi)\right) \sqrt{\mu_{k}} \sin (\phi) \mathrm{d} \phi .
$$

For any $\phi \in\left(\varepsilon, \frac{\pi}{2}\right)$, the following formula for $G_{k}\left(\sqrt{\mu_{k}} \cos (\phi)\right)$ holds (see [29, Theorem 8.22 .9 , p. 201])

$$
\begin{aligned}
& G_{k}\left(\sqrt{\mu_{k}} \cos (\phi)\right)= \\
& \quad \frac{2^{1 / 4}}{\sqrt{\pi}(k-1)^{1 / 4} \sqrt{\sin (\phi)}}\left(\sin \left(\left(\frac{k}{2}-\frac{1}{4}\right)(\sin (2 \phi)-2 \phi)+\frac{3 \pi}{4}\right)+r_{\varepsilon, k}\right),
\end{aligned}
$$

where $r_{\varepsilon, k}$ only depends on $\varepsilon$ and $k$ and, for $\varepsilon$ fixed, $r_{\varepsilon, k}=\underset{k \rightarrow+\infty}{O}\left(\frac{1}{k}\right)$.

Thus,

$$
\begin{aligned}
\int_{\Omega_{n}} G_{k}^{2}(x) \mathrm{d} x & \geq \frac{2 \sqrt{2} \sqrt{2 k-1}}{\pi \sqrt{k-1}} \int_{\varepsilon}^{\frac{\pi}{2}}\left(\sin \left(\left(\frac{k}{2}-\frac{1}{4}\right)(\sin (2 \phi)-2 \phi)+\frac{3 \pi}{4}\right)+r_{\varepsilon, k}\right)^{2} \mathrm{~d} \phi \\
& \geq \frac{4}{\pi} \int_{\varepsilon}^{\frac{\pi}{2}}\left(\sin \left(\left(\frac{k}{2}-\frac{1}{4}\right)(\sin (2 \phi)-2 \phi)+\frac{3 \pi}{4}\right)+r_{\varepsilon, k}\right)^{2} \mathrm{~d} \phi .
\end{aligned}
$$


Let us focus on the term that does not involve $r_{\varepsilon, k}$. Let

$$
v_{k}: \phi \mapsto\left(k-\frac{1}{2}\right)(\sin (2 \phi)-2 \phi)+\frac{3 \pi}{2} .
$$

We have

$$
\begin{aligned}
\frac{4}{\pi} \int_{\varepsilon}^{\frac{\pi}{2}} \sin ^{2}\left(\frac{v_{k}(\phi)}{2}\right) \mathrm{d} \phi & =\frac{2}{\pi} 1-\frac{2}{\pi} \int_{\varepsilon}^{\frac{\pi}{2}} \cos \left(v_{k}(\phi)\right) \mathrm{d} \phi \\
& =1-\varepsilon \frac{2}{\pi}-\frac{2}{\pi} \int_{\varepsilon}^{\frac{\pi}{2}} \cos \left(v_{k}(\phi)\right) \mathrm{d} \phi .
\end{aligned}
$$

To estimate this term, we will fix $\varepsilon$ sufficiently small (depending on $\delta$ ) and prove that the remaining integral goes to 0 when $k$ goes to $+\infty$.

Indeed, integrating by parts, we obtain

$$
\begin{aligned}
\frac{4}{\pi} \int_{\varepsilon}^{\frac{\pi}{2}} \sin ^{2}\left(2 v_{k}(\phi)\right) \mathrm{d} \phi & =1-\varepsilon \frac{2}{\pi}-\frac{2}{\pi} \int_{\varepsilon}^{\frac{\pi}{2}} \cos \left(v_{k}(\phi)\right) \frac{v_{k}^{\prime}(\phi)}{v_{k}^{\prime}(\phi)} \mathrm{d} \phi \\
& =1-\varepsilon \frac{2}{\pi}-\frac{2}{\pi}\left[\frac{\sin \left(v_{k}(\phi)\right.}{v_{k}^{\prime}(\phi)}\right]_{\varepsilon}^{\frac{\pi}{2}} \frac{\sin \left(v_{k}(\phi)\right) v_{k}^{\prime \prime}(\phi)}{\left(v_{k}^{\prime}(\phi)\right)^{2}} \mathrm{~d} \phi .
\end{aligned}
$$

Since, $\sin \left(v_{k}\left(\frac{\pi}{2}\right)\right)=0$ and

$$
\frac{v_{k}^{\prime \prime}(\phi)}{\left(v_{k}^{\prime}(\phi)\right)^{2}}=\frac{-1}{2 k-1} \frac{2 \sin (2 \phi)}{(1-\cos (2 \phi))^{2}}=\frac{-1}{2 k-1} \frac{\cos (\phi)}{\sin ^{3}(\phi)},
$$

we obtain

$$
\frac{4}{\pi} \int_{\varepsilon}^{\frac{\pi}{2}} \sin ^{2}\left(\frac{v_{k}(\phi)}{2}\right) \mathrm{d} \phi \geq 1-\varepsilon \frac{2}{\pi}-\frac{2}{\pi} \frac{1}{1-\cos (2 \varepsilon)} \frac{1}{2 k-1}-\frac{1}{\sin ^{3}(\varepsilon)} \frac{1}{2 k-1} .
$$

Now let us fix $\varepsilon>0$ such that $\varepsilon \frac{2}{\pi}=\frac{\delta}{3}$. There exists $\tilde{K} \in \mathbb{N}^{*}$ such that

$$
\frac{2}{\pi} \frac{1}{1-\cos (2 \varepsilon)} \frac{1}{2 k-1}-\frac{1}{\sin ^{3}(\varepsilon)} \frac{1}{2 k-1} \leq \frac{\delta}{3}, \quad \forall k \geq \tilde{K} .
$$

Notice that $\tilde{K}$ depends only on $\varepsilon$ and thus only on $\delta$.

Finally, let us deal with the remaining terms in (5.7). As $\varepsilon$ is fixed and $r_{\varepsilon, k}=$ $\underset{k \rightarrow+\infty}{O}\left(\frac{1}{k}\right)$, it comes that, there exists $K_{\delta} \in \mathbb{N}^{*}$ depending only on $\delta$ such that

$$
\frac{8}{\pi} r_{\varepsilon, k} \int_{\varepsilon}^{\frac{\pi}{2}} \sin \left(\frac{v_{k}(\phi)}{2}\right) \mathrm{d} \phi+\frac{4}{\pi}\left(\frac{\pi}{2}-\varepsilon\right) r_{\varepsilon, k}^{2} \leq \frac{\delta}{3}, \quad \forall k \geq K_{\delta} .
$$

Using this estimate and (5.8) in (5.7), we obtain that there exists $K \in \mathbb{N}^{*}$ depending only on $\delta$ such that

$$
\int_{\Omega_{n}} G_{k}^{2}(x) \mathrm{d} x \geq 1-\delta, \quad \forall n, k \in \mathbb{N}^{*} \text { such that } \frac{\pi}{2} n \geq k \geq K .
$$

Considering the finite number of $k \in \llbracket 1, K-1 \rrbracket$, as $\left\|G_{k}\right\|_{L^{2}(\mathbb{R})}=1$, it comes that

$$
\int_{\Omega_{n}} G_{k}^{2}(x) \mathrm{d} x \underset{n \rightarrow+\infty}{\rightarrow} 1 .
$$


As $K$ depends only on $\delta$, we deduce that there exists $n_{0}$ depending only on $\delta$ such that

$$
\int_{\Omega_{n}} G_{k}^{2}(x) \mathrm{d} x \geq 1-\delta, \quad \forall k \leq \frac{\pi}{2} n, \forall n \geq n_{0},
$$

which is exactly the generalized statement of Lemma 2.2 announced at the beginning of Section 5.2

\section{REFERENCES}

[1] D. Allonsius. Study of spectrum of Sturm-Liouville operators and applications in controllability of discretized and continuous parabolic problems. Phd thesis, Aix Marseille Université, Sept. 2018. https://hal.archives-ouvertes.fr/tel-01887023

[2] D. Allonsius, F. Boyer, and M. Morancey. Spectral analysis of discrete elliptic operators and applications in control theory. Numer. Math., 140(4):857-911, 2018.

[3] F. Ammar-Khodja, A. Benabdallah, M. González-Burgos, and L. de Teresa. The Kalman condition for the boundary controllability of coupled parabolic systems. Bounds on biorthogonal families to complex matrix exponentials. J. Math. Pures Appl. (9), 96(6):555-590, 2011.

[4] F. Ammar Khodja, A. Benabdallah, M. González-Burgos, and L. de Teresa. Minimal time for the null controllability of parabolic systems: the effect of the condensation index of complex sequences. J. Funct. Anal., 267(7):2077-2151, 2014.

[5] F. Ammar Khodja, A. Benabdallah, M. González-Burgos, and M. Morancey. Quantitative Fattorini-Hautus test and minimal null control time for parabolic problems. J. Math. Pures Appl. (9), 122:198-234, 2019.

[6] K. Beauchard, P. Cannarsa, and R. Guglielmi. Null controllability of Grushin-type operators in dimension two. J. Eur. Math. Soc. (JEMS), 16(1):67-101, 2014.

[7] K. Beauchard, J. Dardé, and S. Ervedoza. Minimal time issues for the observability of Grushintype equations. working paper or preprint, Jan. 2018.

[8] K. Beauchard, L. Miller, and M. Morancey. 2D Grushin-type equations: minimal time and null controllable data. J. Differential Equations, 259(11):5813-5845, 2015.

[9] A. Benabdallah, F. Boyer, M. González-Burgos, and G. Olive. Sharp estimates of the onedimensional boundary control cost for parabolic systems and application to the $\$ \mathrm{n} \$$ dimensional boundary null controllability in cylindrical domains. SIAM Journal on Control and Optimization, 52(5):2970-3001, jan 2014.

[10] A. Benabdallah, F. Boyer, and M. Morancey. A block moment method to handle spectral condensation phenomenon in parabolic control problems. Annales Henri Lebesgue, 2020. to appear.

[11] G. Birkhoff and G. Rota. Ordinary differential equations. Introductions to higher mathematics. Ginn, 1962.

[12] P. Borwein and T. Erdélyi. Polynomials and Polynomial Inequalities. Springer New York, 1995.

[13] P. Borwein and T. Erdélyi. Generalizations of Müntz's theorem via a Remez-type inequality for Müntz spaces. Journal of the American Mathematical Society, 10(02):327-350, apr 1997.

[14] F. Boyer. Controllability of linear parabolic equations and systems, 2020. Lecture Notes, https://hal.archives-ouvertes.fr/hal-02470625

[15] P. Cannarsa, P. Martinez, and J. Vancostenoble. Precise estimates for biorthogonal families under asymptotic gap conditions. Discrete and Continuous Dynamical Systems - Series $S, 2018$.

[16] N. Cîndea, S. Micu, I. Rovenţa, and M. Tucsnak. Particle supported control of a fluid-particle system. J. Math. Pures Appl. (9), 104(2):311-353, 2015.

[17] R. Dautray and J.-L. Lions. Mathematical analysis and numerical methods for science and technology. Vol. 3. Springer-Verlag, Berlin, 1990. Spectral theory and applications, With the collaboration of Michel Artola and Michel Cessenat, Translated from the French by John C. Amson.

[18] M. Duprez and A. Koenig. Control of the Grushin equation: non-rectangular control region and minimal time. ESAIM COCV, 2019. to appear.

[19] T. Duyckaerts and L. Miller. Resolvent conditions for the control of parabolic equations. J. Funct. Anal., 263(11):3641-3673, 2012.

[20] H. O. Fattorini and D. L. Russell. Exact controllability theorems for linear parabolic equations 
in one space dimension. Archive for Rational Mechanics and Analysis, 43(4):272-292, Jan 1971.

[21] H. O. Fattorini and D. L. Russell. Uniform bounds on biorthogonal functions for real exponentials with an application to the control theory of parabolic equations. Quart. Appl. Math., 32:45-69, 1974/75.

[22] G. Fix. Asymptotic eigenvalues of Sturm-Liouville systems. J. Math. Anal. Appl., 19:519-525, 1967.

[23] M. González Burgos and L. Ouaili. Sharp estimates of the one-dimensional boundary control cost for parabolic systems. to appear in Contemp. Math., Amer. Math. Soc., Providence, RI, 2020., 2019.

[24] B. Harris. Asymptotics of eigenvalues for regular sturm-liouville problems. Journal of Mathematical Analysis and Applications, 183(1):25 - 36, 1994.

[25] A. Koenig. Non-null-controllability of the Grushin operator in 2D. Comptes Rendus Mathématique, 355(12):1215 - 1235, Dec. 2017.

[26] J. Lagnese. Control of wave processes with distributed controls supported on a subregion. SIAM J. Control Optim., 21(1):68-85, 1983.

[27] J. Pöschel and E. Trubowitz. Inverse spectral theory, volume 130 of Pure and Applied Mathematics. Academic Press, Inc., Boston, MA, 1987.

[28] L. Schwartz. Étude des sommes d'exponentielles réelles. NUMDAM, Publications de l'Institut de Mathématique de l'Université de Clermont-Ferrand, 1943.

[29] G. Szegó. Orthogonal polynomials. American Mathematical Society, Providence, R.I., fourth edition, 1975. American Mathematical Society, Colloquium Publications, Vol. XXIII.

[30] A. Zettl. Sturm-Liouville theory, volume 121 of Mathematical Surveys and Monographs. American Mathematical Society, Providence, RI, 2005. 